\title{
Tooth enamel microstructure of Revueltosaurus and Krzyzanowskisaurus (Reptilia:Archosauria) from the Upper Triassic Chinle Group, USA: Implications for function, growth, and phylogeny
}

\author{
Andrew B. Heckert and Jessica A. Miller-Camp
}

\begin{abstract}
Tooth enamel microstructure can carry significant phylogenetic, ontogenetic, and functional information within amniotes. Here we provide the first descriptions of the tooth enamel microstructure of two Late Triassic taxa, the crurotarsan Revueltosaurus callenderi Hunt and the putative ornithischian Krzyzanowskisaurus hunti (Heckert), which some consider closely related. To test the hypotheses that enamel thickness corresponds to function and/or phylogeny we analyzed the enamel of each at various scales, measuring enamel thickness and examining microstructural features throughout both longitudinal and cross-sectional thickness using previously established techniques to facilitate comparisons. Both taxa possess thick (up to $\sim 150 \mu \mathrm{m}$ ) enamel for their size $(<20 \mathrm{~mm}$ crown height). Enamel in $R$. callenderi ranged from $\sim 5-152 \mu \mathrm{m}$ across a premaxillary tooth in longitudinal section, and $\sim 42-92 \mu \mathrm{m}$ in a maxillary/dentary tooth transverse section. $K$. hunti enamel thickness was $\sim 18-155 \mu \mathrm{m}$ longitudinally and $\sim 29-75 \mu \mathrm{m}$ transversely. Both also had well-developed basal unit layers (BUL) and weakly developed columnar microstructure. Well-developed lines of incremental growth (LIG) are present in both taxa, through which the columnar enamel grades into parallel crystallite enamel. Their enamel microstructure is therefore grossly similar to that of several ornithischian taxa, especially ankylosaurs, with which they are strongly convergent, and also compares well to rauisuchids and tyrannosaurids. The relatively unique combination of microstructural characteristics in the schmelzmuster of $R$. callenderi and $K$. hunti supports the hypothesis that they are closely related, but does not conclusively preclude a different taxonomic placement for $K$. hunti so we retain its separate generic designation.
\end{abstract}

Andrew B. Heckert. Department of Geology, Appalachian State University, ASU Box 32067, Boone, North Carolina 28608-2067, U.S.A.

Jessica A. Miller-Camp. Department of Geology, Appalachian State University, ASU Box 32067, Boone, North Carolina 28608-2067, U.S.A.

and Department of Geoscience, 121 Trowbridge Hall, University of lowa, lowa City, lowa, 52242, U.S.A.

KEYWORDS: archosaur; tooth enamel; microstructure; Triassic; Revueltosaurus; crurotarsan

PE Article Number: 16.1.1A

Copyright: Society for Vertebrate Paleontology January 2013

Submission: 27 July 2012. Acceptance: 6 December 2012

Heckert, Andrew B. and Miller-Camp, Jessica A. 2013. Tooth enamel microstructure of Revueltosaurus and Krzyzanowskisaurus (Reptilia:Archosauria) from the Upper Triassic Chinle Group, USA: Implications for function, growth, and phylogeny. Palaeontologia Electronica Vol. 16, Issue 1; 1A,23p;

palaeo-electronica.org/content/2013/344-revueltosaurus-tooth-enamel 


\section{INTRODUCTION}

Studies of diapsid tooth enamel microstructure are in their infancy relative to those of synapsids, yet have already yielded insight into amniote evolution ranging from functional interpretations to documenting convergent evolution at multiple levels (Sander, 1999; Hwang, 2005). Within this framework, we examined the enamel microstructure of two teeth each of the unusual crurotarsan Revueltosaurus callenderi Hunt, 1989 and the putative ornithischian Krzyzanowskisaurus hunti (Heckert, 2002); both archosaurs are known from Upper Triassic strata in the American Southwest (Figure 1). Revueltosaurus is a classic example of how much, or how little, fossil teeth can reveal regarding a reptile (non-synapsid amniote). When it was known solely from its teeth, Revueltosaurus callenderi was variously considered a prosauropod (Hunt, 1988), an ?ornithischian (Hunt, 1989), an ornithischian "form genus" (Padian, 1990), Ornithischia incertae sedis and/or a nomen dubium (Sereno, 1991; Norman et al., 2004) and a valid ornithischian taxon (Hunt and Lucas, 1994; Hunt et al., 1998; Heckert, 2002). Upon the discovery of more complete material, including skulls and postcrania, Parker et al. (2005) demonstrated that, although the teeth are diagnostic, $R$. callenderi is actually a crurotarsan archosaur. The present study on the microstructure of $R$. callenderi was first undertaken when Revueltosaurus was considered a basal ornithischian, as all of the ornithischian taxa sampled in the definitive treatment of reptilian enamel microstructure (Sander, 1999) were both young (Early Cretaceous or younger) and relatively derived (e.g., Iguanodon). Thus, this study was originally designed to help fill in that gap. Now that Revueltosaurus has been found to occupy a very different branch of the archosaurian tree, we feel that there is still something to learn from documenting these structures, both to complement and expand upon Sander's (1999) work and to provide basic morphological information on this unusual taxon. Similarly, Krzyzanowskisaurus was originally described as Revueltosaurus hunti (Heckert, 2002). Heckert (2005) assigned it to its own genus and considered it an ornithischian (Heckert, 2002) but Parker et al. (2005) considered it to likely represent a Revueltosaurus-like crurotarsan (Figure 2).

Anatomical abbreviations: $\mathrm{BUL}=$ basal unit layer; $E D J$ = enamel-dentine junction; LIG = lines of incremental growth (incremental lines of some authors); OES = outer enamel surface.
Institutional abbreviations: $\mathrm{NMMNH}=\mathrm{New}$ Mexico Museum of Natural History and Science, Albuquerque; UCMP = University of California Museum of Paleontology, Berkeley.

\section{MATERIALS AND METHODS}

We sectioned two teeth of Revueltosaurus callenderi from its type locality (NMMNH locality 1 ) in the Bull Canyon Formation of eastern New Mexico. These teeth include both a premaxillary (NMMNH P-33799) and a maxillary/dentary (NMMNH P-33798) tooth, as first hypothesized by Hunt (1989) and confirmed by Parker et al. (2005) with more complete materials. The teeth were embedded in epoxy and sectioned at the Steinmann-Institut für Geologie, Mineralogie und Paläontologie, Bereich Paläontology, then called the Institut für Paläontologie, Universität Bonn, using techniques similar to that utilized by Sander (1999) in his previous studies (Sander, personal commun. 2005). The premaxillary tooth was sectioned longitudinally and the maxillary tooth was sectioned transversely. We also sectioned two teeth of Krzyzanowskisaurus hunti from the Petrified Forest Formation of eastern Arizona using similar protocols as described by Hwang (2005). We sectioned UCMP 165213 in longitudinal section and UCMP 165211 in transverse section. All teeth were etched for 30 seconds in $5 \% \mathrm{HCl}$. They were then cleaned in an ultrasonic bath for 30 seconds before being coated with gold in a sputter coater. The scanning electron microscope (SEM) images here were taken using a Quanta 200 ESEM utilizing XT microscope server imaging software housed at the College of Arts and Sciences microscopy facility at Appalachian State University.

We note that tooth position is harder to establish in Krzyzanowskisaurus than Revueltosaurus, as most Krzyzanowskisaurus teeth resemble more complex versions of Revueltosaurus premaxillary teeth. Heckert $(2002,2005)$ hypothesized that the taller, less symmetrical tooth crowns of Krzyzanowskisaurus lacking additional cingula are probably premaxillary teeth, and that the more complex teeth with labial and lingual cingula likely represent maxillary/dentary teeth. However, it is also possible that these doubly cingulated teeth wore against an outer, rhamphotheca-like (beak) structure. Regardless, according to this hypothesis UCMP 165213 may represent a premaxillary tooth, and UCMP 165211 may represent a maxillary/dentary tooth. Accordingly, we sectioned them longitudinally and transversely, respectively, to facilitate comparisons with Revueltosaurus. These teeth 


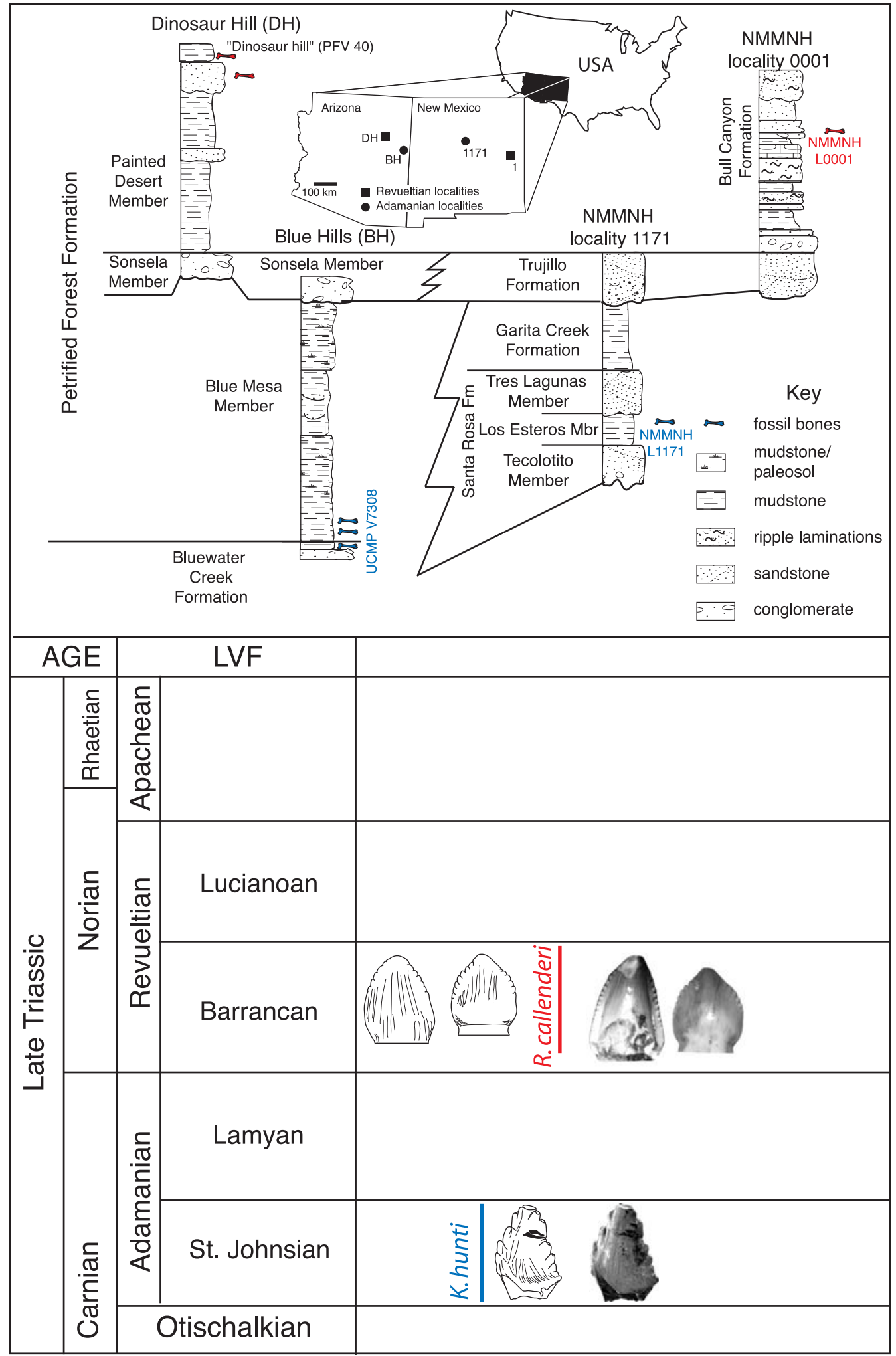

FIGURE 1. Location map and stratigraphic section showing the geographic and stratigraphic distribution of Revueltosaurus and Krzyzanowskisaurus. This includes the localites of the Revueltosaurus (NMMNH locality 1) and Krzyzanowskisaurus (UCMP locality 7307) teeth described here. Tooth illustrations after Heckert $(2002,2005)$. LVF = land vertebrate faunachron (following Lucas et al., 2007); L-1171 = type location of Krzyzanowskisaurus hunti; PFV = locality yielding abundant Revueltosaurus fossils in the Petrified Forest National Park. 


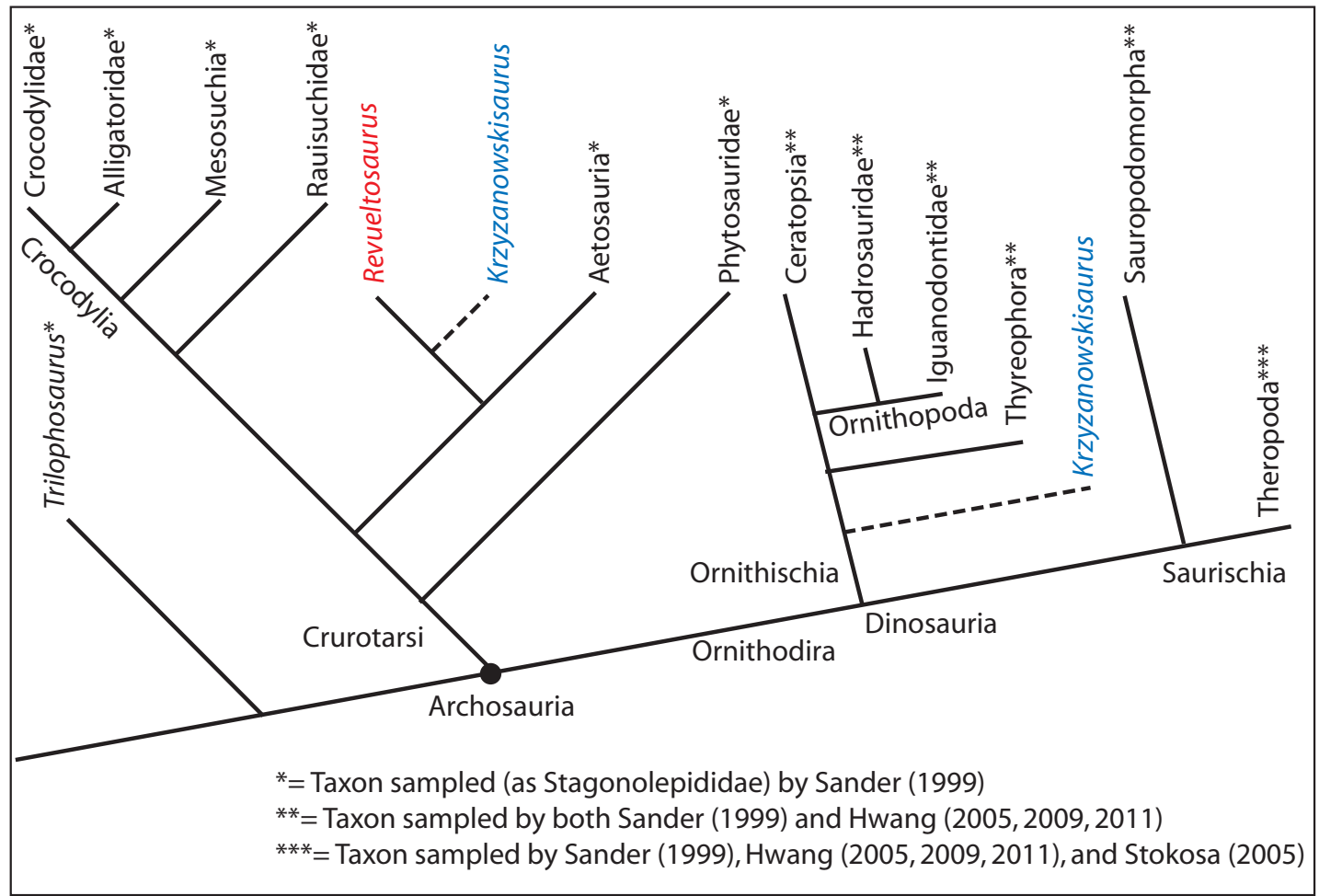

FIGURE 2. Generalized archosaurian phylogeny showing the relationships of taxa sampled by Sander (1999) and Hwang (2005) (in black) as well as Revueltosaurus (blue) and Krzyzanowskisaurus (red). Dashed lines demonstrate the two hypothesized positions of Krzyzanowskisaurus, either as a crurotarsan closely allied to Revueltosaurus (e.g., Parker et al., 2005; Irmis et al., 2007) or a basal ornithischian (Heckert, 2002, 2005).

were not as well preserved, and thus were less amenable to sectioning and SEM examination, resulting in fewer images and measurements, although we note that there is no reason not to consider the available images and measurements representative.

The tooth enamel microstructure terminology we utilize reflects that developed in previous studies, principally Sander $(1999,2000)$ and Hwang (2005), and the macroscopic tooth descriptions follow Smith and Dodson (2003) generally and previous descriptions of Revueltosaurus teeth (e.g., Hunt, 1989; Hunt and Lucas, 1994; Heckert, 2002) in particular. We note that this means that we use mesial/distal, labial/lingual, and basal/apical (or occlusal) to describe and orient isolated teeth and features of the tooth crown. Descriptions of microstructural features follow the standardized format of Hwang (2005, 2011), with enamel described from the enamel-dentine junction (EDJ) outward to the outer enamel surface (OES). Enamel thickness and distribution is described first, followed by type and schmelzmuster, followed by discussion of any special features. Particular features we describe are the basal unit layer (BUL) and lines of incre- mental growth (LIG). We use the term "lines of incremental growth" (LIG) here, as used by Hwang (2005) to describe interruptions in the microstructural texture. These are the "incremental lines" of Sander (1999) and Stokosa (2005) and are also known as "striae of Retzius" in mammalian/human anatomical terminology. Presently it is unclear what governs the growth cycles of LIGs in reptiles, although Appenzeller et al. (2005) recently argued that they are developed daily and represent the activity of the autonomous nervous system.

We calculated the enamel thicknesses reported here by measuring their thickness perpendicular to the enamel-dentine junction in the images used here, using the program tpsDig 2.0 (Rohlf, 2010). Although tpsDig provided up to five significant figures (to hundredth of a micron), for a variety of reasons we suggest using no more than three. We recognize the difficulty of replicating measurements, especially if the section images are not perfectly perpendicular to the electron gun, and so made 10 measurements (close together) on one image to assess the precision of the tpsDig measurement protocol. After accepting the mean of these measurements as the "true value" (and the 
number reported in the tables), we calculated the absolute value of the difference between measured and "true" values, and divided the mean of that difference by the "true value." We used a similar procedure for the median value, and both the mean and median values provide a relative error of $0.5 \%$ (0.005), so we consider these measurements the most precise estimates of sauropsid enamel thickness in the literature. Obviously, slightly oblique sections will inflate thickness measurements, so the measurements we provide, although more precise than any previously reported for archosaurian tooth enamel microstructure, should be considered as maximum estimates.

\section{HISTORY OF STUDY}

There are two distinct aspects of the history of this study, one regarding the study of Revueltosaurus and Krzyzanowskisaurus and another related to the study of reptilian tooth enamel microstructure.

Regarding the first aspect, Hunt (1989) named Revueltosaurus callenderi as an ?ornithischian dinosaur. Various authors reported additional occurrences and noted its biostratigraphic utility (e.g., Padian, 1990; Hunt and Lucas, 1994; Long and Murry, 1995; Heckert and Lucas, 1997; Hunt et al., 1998). Although there was some debate regarding the validity of the taxon, most workers accepted it as a likely basal ornithischian albeit a nomen dubium (e.g., Sereno, 1991; Norman et al., 2004). Heckert (2002) reviewed Revueltosaurus occurrences and recognized a second species, Revueltosaurus hunti for older, more complex teeth originally assigned to Revueltosaurus callenderi by Long and Murry (1995). Parker et al. (2005; see also Hunt et al., 2005) reported postcrania associated with tooth-bearing elements demonstrating that $R$. callenderi was a crurotarsan, not a dinosaur, and Heckert (2005) coined the new generic name Krzyzanowskisaurus for "R." hunti. The affinities of the latter remain enigmatic, with Parker et al. (2005) positing that it likely represents a crurotarsan allied to R. callenderi, and Heckert (2005) hypothesizing that the more derived dentition of Krzyzanowskisaurus really is that of an ornithischian. Irmis et al. (2007; see also Nesbitt et al., 2007) interpreted the "cingulum" of Krzyzanowskisaurus hunti to represent an autapomorphic state, but otherwise considered the tooth morphology similar enough to justify retention of " $K$." hunti in Revueltosaurus as $R$. hunti To date, unambiguous Krzyzanowskisaurus remains are known only from teeth, so we chose to investigate the tooth enamel microstructure of Revueltosaurus and Krzyzanowskisaurus as an additional means of assessing the degree of similarity between the two taxa.

"Reptilian" (sauropsid) tooth enamel microstructure studies were not truly feasible until the advent of SEM microscopy (see Sander, 1999 for a more complete review). Accordingly, the study of archosaurian tooth enamel has a history of study nearly as brief as the taxonomic history of Revueltosaurus and Krzyzanowskisaurus. Buffetaut et al. (1986) were the first to publish SEM images of dinosaurian tooth enamel microstructure, and additional studies led by Dauphin (Dauphin, 1988, Dauphin et al., 1988a,b) were published shortly thereafter. These later studies sampled a variety of dinosaurian and non-dinosaurian archosaurs, among others, and in these taxa the enamel microstructure was described in general terms. Following the monumental work on synapsid enamel microstructure edited by Koenigswald and Sander (1997), Sander $(1999,2000)$ published the first reasonably systematic survey of sauropsid tooth enamel microstructure. His work, especially the 1999 monograph, remains the standard reference for microstructural features in non-synapsid amniotes. More recently Stokosa $(2001,2005)$ examined the microstructure of a variety of tyrannosaurid and dromaeosaurid taxa from the Upper Cretaceous of western North America, and Hwang (2005, 2006, 2007, 2009, 2011) sampled an array of dinosaurian teeth from the Mesozoic of North America as well as the Cretaceous of Asia en route to completing a dissertation (Hwang, 2007) and related abstracts and papers (Hwang, 2005, 2006, 2009, 2011). Unlike Sander (1999), who saw little phylogenetic signal in enamel microstructure, especially at lower (family-level) taxonomic rank, Hwang $(2005,2009)$ identified some similarity in tooth enamel microstructures related to phylogenetic position, although she concurs with Sander (1999) that relatively few unambiguous synapomorphies are evident in the enamel microstructure at lower taxonomic levels. In Table 1 we summarize many of the observations of Sander (1999), Hwang (2005, 2009), and Stokosa (2005) as well as our interpretations of the results of earlier workers based on their descriptions and published illustrations. This study supersedes an extended abstract we published previously that focused solely on Revueltosaurus (Heckert and Camp, 2006). 
TABLE 1. Comparison of tooth enamel thickness and enamel microstructural features in different archosauromorphs compiled from the literature.

Tooth Enamel Microstructure

\begin{tabular}{|c|c|c|c|c|c|c|c|c|c|c|c|}
\hline Higher Taxon & Taxon & Sampler & 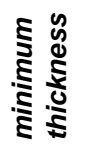 & 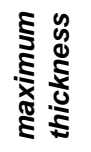 & 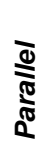 & $\frac{\hbar}{\stackrel{\varpi}{\Sigma}}$ & క্̉ & $\stackrel{0}{9}$ & 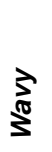 & $\underset{\mathfrak{D}}{\stackrel{\varrho}{S}}$ & Comments \\
\hline Archosauromorpha & Trilophosaurus & Sander (1999) & 20 & 20 & & & $x$ & $x$ & & & \\
\hline \multirow[t]{4}{*}{ Crurotarsi } & $\begin{array}{l}\text { Phytosauridae } \\
\text { (Dockum) }\end{array}$ & Sander (1999) & 20 & 20 & $x$ & & & $x$ & & & I and III \\
\hline & $\begin{array}{l}\text { Phytosauridae } \\
\text { (Dockum) }\end{array}$ & Sander (1999) & & 150 & $x$ & $x$ & $x$ & $x$ & & & II \\
\hline & Phytosauridae (Hallau) & )Sander (1999) & & 60 & & $x$ & & & & & \\
\hline & Rauisuchidae & Sander (1999) & 60 & 100 & $x$ & $x$ & $x$ & $x$ & & & $\begin{array}{l}95 \% \text { columnar; } \\
\text { outer is parallel; } \\
\text { rare LIG }\end{array}$ \\
\hline Mesosuchia & Machimosaurus hugi & Sander (1999) & & 350 & $x$ & $x$ & $x$ & & & & LIG rare \\
\hline \multirow[t]{2}{*}{$\begin{array}{l}\text { Crocodylia: } \\
\text { Alligatoridae }\end{array}$} & Allognathosuchus sp. & Sander (1999) & & 300 & $x$ & $x$ & & $x$ & & & $\begin{array}{l}\text { up to } 50 / 50 \\
\text { columnar/parallel; } \\
\text { sometimes } 95 / 5 \text {; } \\
\text { LIG only in parallel }\end{array}$ \\
\hline & $\begin{array}{c}\text { Alligator } \\
\text { mississippiensis }\end{array}$ & Sander (1999) & & 1000 & $x$ & $x$ & $x$ & $x$ & & & $\begin{array}{l}\text { Mostly columnar; } \\
\text { LIG in columnar } \\
\text { enamel }\end{array}$ \\
\hline \multirow[t]{4}{*}{$\begin{array}{l}\text { Crocodylia: } \\
\text { Crocodylidae }\end{array}$} & $\begin{array}{l}\text { Deinosuchus } \\
\text { riograndensis }\end{array}$ & Sander (1999) & $<150$ & 525 & & $x$ & $x$ & & & & $\begin{array}{l}40 \% \text { columnar; } \\
55 \% \text { microunit; only } \\
\text { specimen w/ } \\
\text { described } \\
\text { microunits }\end{array}$ \\
\hline & Asiatosuchus & Sander (1999) & & 500 & & $x$ & $x$ & & & & $\begin{array}{l}\text { compound unit } \\
\text { enamel/weakly } \\
\text { columnar }\end{array}$ \\
\hline & Pristichampsa & Sander (1999) & 20 & 50 & $x$ & $x$ & & $x$ & & & $\begin{array}{l}\text { LIG best in the } \\
\text { carinae }\end{array}$ \\
\hline & Eusuchia indet & Sander (1999) & 50 & 125 & $x$ & $x$ & & $x$ & & & $\begin{array}{l}\text { Columnar yields to } \\
\text { parallel }\end{array}$ \\
\hline \multicolumn{12}{|c|}{ Dinosauria:Saurischia: Theropoda } \\
\hline & $\begin{array}{l}\text { Basal Theropoda } \\
\text { indet. }\end{array}$ & Hwang (2009) & & & $x$ & $x$ & $x$ & & & $x$ & $\begin{array}{l}100 \mu \mathrm{m} \text { average } \\
\text { thickness; Kayenta } \\
\text { Fm "carnosaur" in } \\
\text { UCMP collections }\end{array}$ \\
\hline \multirow[t]{3}{*}{ D:S:T:Ceratosauria } & Coelophysis bauri & $\begin{array}{l}\text { Hwang }(2005, \\
\text { 2011) }\end{array}$ & 10 & 10 & $x$ & & & $x$ & & & $\begin{array}{l}\text { C. bauri of Hwang } \\
\text { (2005) a distinct } \\
\text { taxon (Hwang, } \\
2011, \text { p. 192) }\end{array}$ \\
\hline & $\begin{array}{l}\text { Ceratosaurus } \\
\text { nasicornis }\end{array}$ & Hwang (2011) & 20 & 70 & $x$ & $x$ & $x$ & $x$ & & $x$ & $\begin{array}{l}120 \mu \mathrm{m} \text { average } \\
\text { thickness; varies } \\
\text { wildly }\end{array}$ \\
\hline & Majungasaurus sp. & Hwang (2011) & $<50$ & $>90$ & $x$ & $x$ & $x$ & $x$ & & $x$ & \\
\hline \multirow[t]{3}{*}{ D:S:T:Allosauroidea } & Allosaurus fragilis & Hwang (2011) & 20 & $>85$ & $x$ & $x$ & $x$ & $x$ & & & \\
\hline & cf. Allosaurus & Sander (1999) & 10 & 15 & $x$ & & & & & & Not even LIG \\
\hline & Charcharodontosaurus & $\begin{array}{l}\text { sBuffetaut et al. } \\
\qquad(1986)\end{array}$ & & & & $x$ & & & & & $\begin{array}{l}\text { called "prisms" in } \\
\text { published } \\
\text { description }\end{array}$ \\
\hline
\end{tabular}


TABLE 1 (continued).

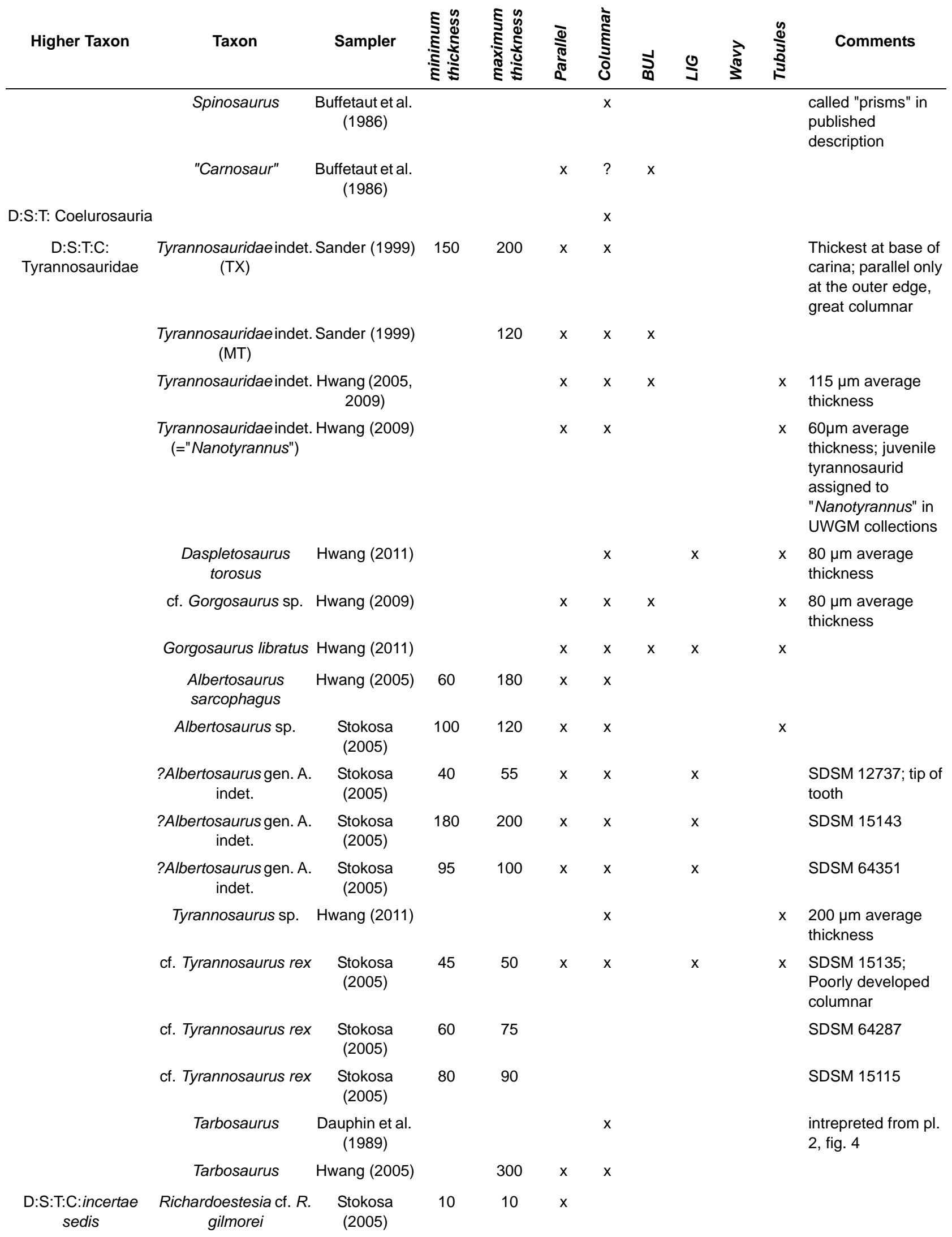


Heckert and Miller-Camp: Revueltosaurus tooth enamel

TABLE 1 (continued).

\begin{tabular}{|c|c|c|c|c|c|c|c|c|c|c|c|}
\hline Higher Taxon & Taxon & Sampler & 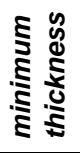 & 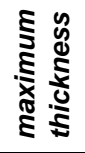 & 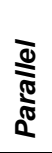 & 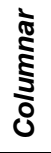 & Ș & $\stackrel{0}{\mathfrak{y}}$ & 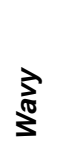 & 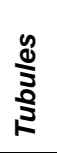 & Comments \\
\hline & $\begin{array}{c}\text { Richardoestesia } \\
\text { gilmorei }\end{array}$ & Hwang (2011) & $\sim 10$ & $\sim 10$ & $x$ & & $x$ & $x$ & & $x$ & $\begin{array}{l}10 \mu \mathrm{m} \text { average } \\
\text { thickness }\end{array}$ \\
\hline & $\begin{array}{l}\text { Richardoestesia } \\
\text { isosceles }\end{array}$ & Hwang (2011) & $\sim 13$ & $\sim 13$ & $x$ & & $x$ & & & $x$ & $\begin{array}{l}13 \mu \mathrm{m} \text { average } \\
\text { thickness }\end{array}$ \\
\hline & Richardoestesia sp. & $\begin{array}{l}\text { Stokosa } \\
(2005)\end{array}$ & 10 & 15 & $x$ & & & & & & \\
\hline \multirow[t]{19}{*}{ D:S:T:C:Maniraptora } & Troodontid indet. A & Hwang (2005) & & 60 & $x$ & & & & & & \\
\hline & Troodontid indet. B & Hwang (2005) & & & $x$ & $x$ & & & & & \\
\hline & $\begin{array}{c}\text { Troodontid n. gen. et. } \\
\text { Sp. }\end{array}$ & Hwang (2005) & & 30 & $x$ & & & $x$ & & & \\
\hline & Troodon sp. & Sander (1999) & & 20 & $x$ & $x$ & $x$ & & & & $\begin{array}{l}\text { Columnar units } \\
\text { small }(2 \mu \mathrm{m}) \text { and } \\
\text { weak, seem to } \\
\text { arise from BUL } \\
\text { (Sander, p. } 65)\end{array}$ \\
\hline & Troodon sp. & $\begin{array}{l}\text { Hwang (2009, } \\
\text { 2011) }\end{array}$ & & & $\mathrm{x}$ & & $x$ & & & & $\begin{array}{l}20 \mu \mathrm{m} \text { average } \\
\text { thickness; parallel } \\
\text { crystallites }\end{array}$ \\
\hline & $\begin{array}{l}\text { Troodon sp. cf. } T \text {. } \\
\text { formosus }\end{array}$ & $\begin{array}{c}\text { Stokosa } \\
(2005)\end{array}$ & 10 & 15 & $x$ & & & & & & \\
\hline & $\begin{array}{l}\text { Paronychodon cf. } P . \\
\text { lacustris }\end{array}$ & $\begin{array}{l}\text { Stokosa } \\
\text { (2005) }\end{array}$ & 0 & 15 & $x$ & & & & & & \\
\hline & $\begin{array}{l}\text { Paronychodon } \\
\text { lacustris }\end{array}$ & Sander (1999) & & 20 & $x$ & & & $x$ & & & $\begin{array}{l}\text { LIG are few and } \\
\text { weak }\end{array}$ \\
\hline & $\begin{array}{l}\text { Paronychodon } \\
\text { (Troodontid) }\end{array}$ & Hwang (2005) & & & $x$ & & & $x$ & & & \\
\hline & Byronosaurus jaffei & Hwang (2005) & & 13 & $x$ & & & $x$ & & & \\
\hline & $\begin{array}{l}\text { Paronychodon } \\
\text { (Dromaeosaurid) }\end{array}$ & Hwang (2005) & & & & & & & & & \\
\hline & Velociraptor & $\begin{array}{l}\text { Dauphin et al. } \\
\text { (1989) }\end{array}$ & & & $x$ & & & & & & $\begin{array}{l}\text { interpreted from pl. } \\
1 \text {, figs. } 4-5\end{array}$ \\
\hline & $\begin{array}{l}\text { Velociraptor } \\
\text { mongoliensis }\end{array}$ & Hwang (2005) & & 24 & $x$ & & $x$ & & & & \\
\hline & Bambiraptor feinbergi & i Hwang (2005) & & & $x$ & & & $x ?$ & & & $\begin{array}{l}\text { No LIG in Hwang } \\
\text { (2005), but faint in } \\
\text { Hwang (writ. } \\
\text { Comm.) }\end{array}$ \\
\hline & $\begin{array}{c}\text { Dromaeosauridae } \\
\text { indet. }\end{array}$ & Hwang (2005) & & 55 & $x$ & & $x$ & & & & \\
\hline & $\begin{array}{l}\text { Deinonychus } \\
\text { antirrhopus }\end{array}$ & Hwang (2011) & 17 & $30+$ & $x$ & & $x$ & $x$ & & $x$ & $\begin{array}{l}\text { BUL poorly } \\
\text { developed; LIG } \\
\text { faint }\end{array}$ \\
\hline & Saurornitholestes sp. & Hwang (2011) & 7 & $20+$ & $x$ & & $x$ & & & $x$ & $\begin{array}{l}\text { BUL half of enamel } \\
\text { thickness }\end{array}$ \\
\hline & Dromaemosaurus sp. & Hwang (2011) & 25 & 35 & $x$ & & $x$ & $x$ & & $\mathrm{x}$ & $\begin{array}{l}\text { BUL half of enamel } \\
\text { thickness, but not } \\
\text { well-developed }\end{array}$ \\
\hline & $\begin{array}{c}\text { Dromaeosaurus sp. cf. } \\
\text { D. albertensis }\end{array}$ & $\begin{array}{l}\text { Stokosa } \\
(2005)\end{array}$ & 40 & 45 & $x$ & $x$ & & $x$ & & & $\begin{array}{l}\text { Columnar at EDJ, } \\
\text { divergent parallel } \\
\text { more at OES }\end{array}$ \\
\hline
\end{tabular}


TABLE 1 (continued).

\begin{tabular}{|c|c|c|c|c|c|c|c|c|c|}
\hline Higher Taxon & Sampler & 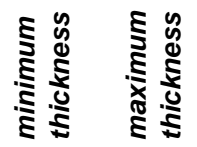 & 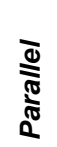 & 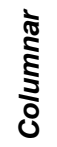 & Ș & $\stackrel{0}{9}$ & 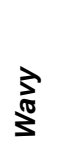 & $\underset{\mathfrak{S}}{\stackrel{0}{\mathfrak{S}}}$ & Comments \\
\hline \multirow[t]{2}{*}{ D:S:T:Avialae } & $\begin{array}{l}\text { Indeterminate Avialan Hwang (2011) } \\
\text { A }\end{array}$ & & $x$ & & $x$ & & & $x$ & $\begin{array}{l}20 \mu \mathrm{m} \text { average } \\
\text { thickness }\end{array}$ \\
\hline & $\begin{array}{l}\text { Indeterminate Avialan Hwang (2011) } \\
\text { B }\end{array}$ & & $x$ & & $x$ & & & $x$ & $\begin{array}{l}16 \mu \mathrm{m} \text { average } \\
\text { thickness }\end{array}$ \\
\hline
\end{tabular}

Sauropodomorpha

\begin{tabular}{|c|c|c|c|c|c|c|c|c|}
\hline & $\begin{array}{l}\text { Plateosaurus } \\
\text { engelhardti }\end{array}$ & Sander (1999) & 10 & 40 & $x$ & $?$ & & $x$ \\
\hline & cf. Diplodocus & Sander (1999) & & 150 & & & $x$ & \\
\hline & Diplodocus longus & Hwang (2011) & 440 & 490 & & $x$ & $x$ & $x$ \\
\hline & Camarasaurus sp. & Hwang (2011) & 700 & $1000+$ & $x$ & $x$ & $x$ & $x$ \\
\hline & Titanosauridae indet. & Hwang (2011) & & & $x$ & $x$ & $x$ & $x$ \\
\hline uria:Ornit & hia & & & & & & & \\
\hline D:O:Stegosauria & Stegosaurus sp. & Hwang (2011) & & & $\mathrm{x}$ & & $x$ & $x$ \\
\hline D:O:Ankylosauria & Ankylosauria indet. & Sander (1999) & & 60 & & $x$ & $x$ & \\
\hline & $\begin{array}{c}\text { Ankylosaurus } \\
\text { magniventris }\end{array}$ & Hwang (2005) & & 60 & $x$ & $x$ & $x$ & $x$ \\
\hline & $\begin{array}{l}\text { Edmontonia } \\
\text { rugosidens }\end{array}$ & Hwang (2005) & & $100+$ & $x$ & $x$ & $x$ & $x$ \\
\hline & Sauropelta edwardsi & Hwang (2005) & & 105 & $x$ & $x$ & $x$ & $\mathrm{x}$ \\
\hline & Euplocephalus & $\begin{array}{l}\text { Hwang (2009, } \\
\text { 2011) }\end{array}$ & 35 & 65 & $x$ & $x$ & $x$ & $x$ \\
\hline
\end{tabular}

Dinosauria:Ornithischia

30-40 $\mu \mathrm{m}$ average thickness

\section{Dinosauria:Ornithischia:Euornithopoda}

\begin{tabular}{|c|c|c|c|c|c|c|c|c|c|c|c|}
\hline \multirow{2}{*}{$\begin{array}{c}\text { D:O:E: } \\
\text { Hypsilophodontidae }\end{array}$} & Thescelosaurus sp. & Sander (1999) & 14 & 140 & $x$ & & $x$ & & & & BUL thin \\
\hline & Thescelosaurus sp. & Hwang (2011) & 20 & 90 & $x$ & $x$ & $x$ & & & $x$ & $\begin{array}{l}\text { Hwang (2011) } \\
\text { suspects Sander's } \\
\text { (1999) specimen is } \\
\text { not Thescelosaurus }\end{array}$ \\
\hline \multirow[t]{2}{*}{ D:O:E:Dryomorpha } & Dryosaurus altus & Hwang (2011) & & & & & $x$ & $\mathrm{x}$ & $x$ & $x$ & $\begin{array}{l}55-65 \mu \mathrm{m} \text { average } \\
\text { thickness }\end{array}$ \\
\hline & Camptosaurus dispar & Hwang (2011) & & & & & $x$ & & $x$ & $x$ & \\
\hline \multirow[t]{2}{*}{$\begin{array}{c}\text { D:O:E: } \\
\text { Iguanodontidae }\end{array}$} & Iguanodon sp. & Sander (1999) & 100 & 150 & & & $x$ & & $x$ & & $\begin{array}{l}\text { Inner and outer } \\
\text { wavy enamel }\end{array}$ \\
\hline & Tenontosaurus tilleti & Hwang (2005) & & $100+$ & $x$ & $\mathrm{x}$ & $\mathrm{x}$ & & & $x$ & \\
\hline \multirow[t]{2}{*}{$\begin{array}{c}\text { D:O:E: } \\
\text { Hadrosauridae }\end{array}$} & Hadrosauridae indet. & Sander (1999) & 160 & 210 & & & $x$ & & $x$ & & \\
\hline & Hadrosaurinae indet. & Hwang (2009) & & & & $x$ & & & & $x$ & \\
\hline
\end{tabular}


Heckert and Miller-Camp: Revueltosaurus tooth enamel

TABLE 1 (continued).

\begin{tabular}{|c|c|c|c|c|c|c|c|c|c|c|c|}
\hline Higher Taxon & Taxon & Sampler & 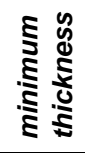 & 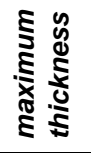 & 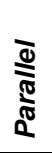 & 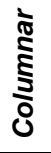 & క్ర & $\stackrel{0}{3}$ & 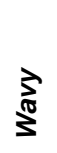 & 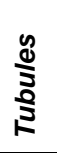 & Comments \\
\hline & Anatosaurus sp. & Sander (1999) & 100 & $100+$ & & & $x$ & & $x$ & $x$ & BUL inverted \\
\hline & Saurolophus sp. & Hwang (2011) & & $165+$ & & & $x$ & & $\mathrm{x}$ & & $\begin{array}{l}115 \mu \mathrm{m} \text { average } \\
\text { thickness }\end{array}$ \\
\hline & $\begin{array}{l}\text { Gilmoreosaurus } \\
\text { mongoliensis }\end{array}$ & Hwang (2005) & & & & & $x$ & & $\mathrm{x}$ & & \\
\hline & Bactrosaurus johnsoni & i Hwang (2005) & & & & & $x$ & & $x$ & & \\
\hline & Kritosaurus navajovius & s Hwang (2005) & & & & & $x$ & & $\mathrm{x}$ & & \\
\hline & $\begin{array}{l}\text { Hypacrosaurus } \\
\text { altispinus }\end{array}$ & Hwang (2005) & & & & & $x$ & & $\mathrm{x}$ & & \\
\hline & $\begin{array}{l}\text { Corythosaurus } \\
\text { casuaris }\end{array}$ & Hwang (2005) & & & & & $x$ & & $x$ & & \\
\hline & $\begin{array}{l}\text { Prosaurolophus } \\
\text { maximus }\end{array}$ & Hwang (2011) & & $\sim 200$ & & & $x$ & & $x$ & & $\begin{array}{l}135 \mu \mathrm{m} \text { average } \\
\text { thickness }\end{array}$ \\
\hline \multicolumn{12}{|c|}{ Dinosauria:Ornithischia: Ceratopsia } \\
\hline & Neoceratopsia indet. & Hwang (2009) & & & & $x$ & & & & & $\begin{array}{l}\text { Average } 285 \mu \mathrm{m} \text {; } \\
\text { originally assigned } \\
\text { to Thescelosaurus } \\
\text { sp. }\end{array}$ \\
\hline & Psittacosaurus sp. & Hwang (2005) & & & & $x$ & $\mathrm{x}$ & & & $?$ & enamel voids \\
\hline & Protoceratops & $\begin{array}{l}\text { Dauphin et al. } \\
\text { (1988) }\end{array}$ & & & $x$ & $x$ & $\mathrm{x}$ & & & & $\begin{array}{l}\text { interpreted from pl. } \\
1 \text {, figs. } 7-8 \text { (maxilla) } \\
\text { and } 12 \text { (premaxilla) }\end{array}$ \\
\hline & Leptoceratops gracilis & Hwang (2005) & & 420 & & $x$ & $x$ & & & $x$ & \\
\hline & Protoceratops sp. & Hwang (2005) & & $120+$ & & $x$ & $x$ & & & $\mathrm{x}$ & \\
\hline \multirow[t]{5}{*}{ D:O:C:Ceratopsidae } & $\begin{array}{l}\text { Ceratopsidae indet. } \\
\text { (Can) }\end{array}$ & Sander (1999) & 150 & & & $x$ & $x$ & $\mathrm{x}$ & & $\mathrm{x}$ & \\
\hline & $\begin{array}{l}\text { Ceratopsidae indet. } \\
\text { (WY) }\end{array}$ & Sander (1999) & & & & & & & & & \\
\hline & Triceratops sp. & Hwang (2005) & & 325 & & $x$ & $x$ & & & $x$ & \\
\hline & Centrosaurus apertus & Hwang (2011) & & & & $x$ & $x$ & $\mathrm{x}$ & & $x$ & $\begin{array}{l}170-270 \mu \mathrm{m} \\
\text { average thickness } \\
\text { depending on } \\
\text { position }\end{array}$ \\
\hline & $\begin{array}{l}\text { Pachyrhinosaurus } \\
\text { canadensis }\end{array}$ & Hwang (2011) & & & & $x$ & $x$ & $x$ & & $x$ & $\begin{array}{l}170-270 \mu \mathrm{m} \\
\text { average thickness } \\
\text { depending on } \\
\text { position }\end{array}$ \\
\hline \multicolumn{12}{|c|}{ Dinosauria:Ornithischia:Pachycephalosauridae } \\
\hline & $\begin{array}{c}\text { Pachycephalosauridae } \\
\text { indet. A }\end{array}$ & Hwang (2005) & & 20 & $x$ & & & & & & $\begin{array}{l}\text { anterior tooth, } \\
\text { diverging parallel }\end{array}$ \\
\hline & $\begin{array}{c}\text { Pachycephalosauridae } \\
\text { indet. B }\end{array}$ & Hwang (2005) & & 40 & $x$ & $x$ & $x$ & & & & $\begin{array}{l}\text { posterior tooth, } \\
\text { incipient columnar }\end{array}$ \\
\hline & $\begin{array}{c}\text { Pachycephalosauridae } \\
\text { indet. C }\end{array}$ & eHwang (2005) & & 50 & $x$ & $x$ & $x$ & & & & $\begin{array}{l}\text { posterior tooth, } \\
\text { incipient columnar }\end{array}$ \\
\hline
\end{tabular}




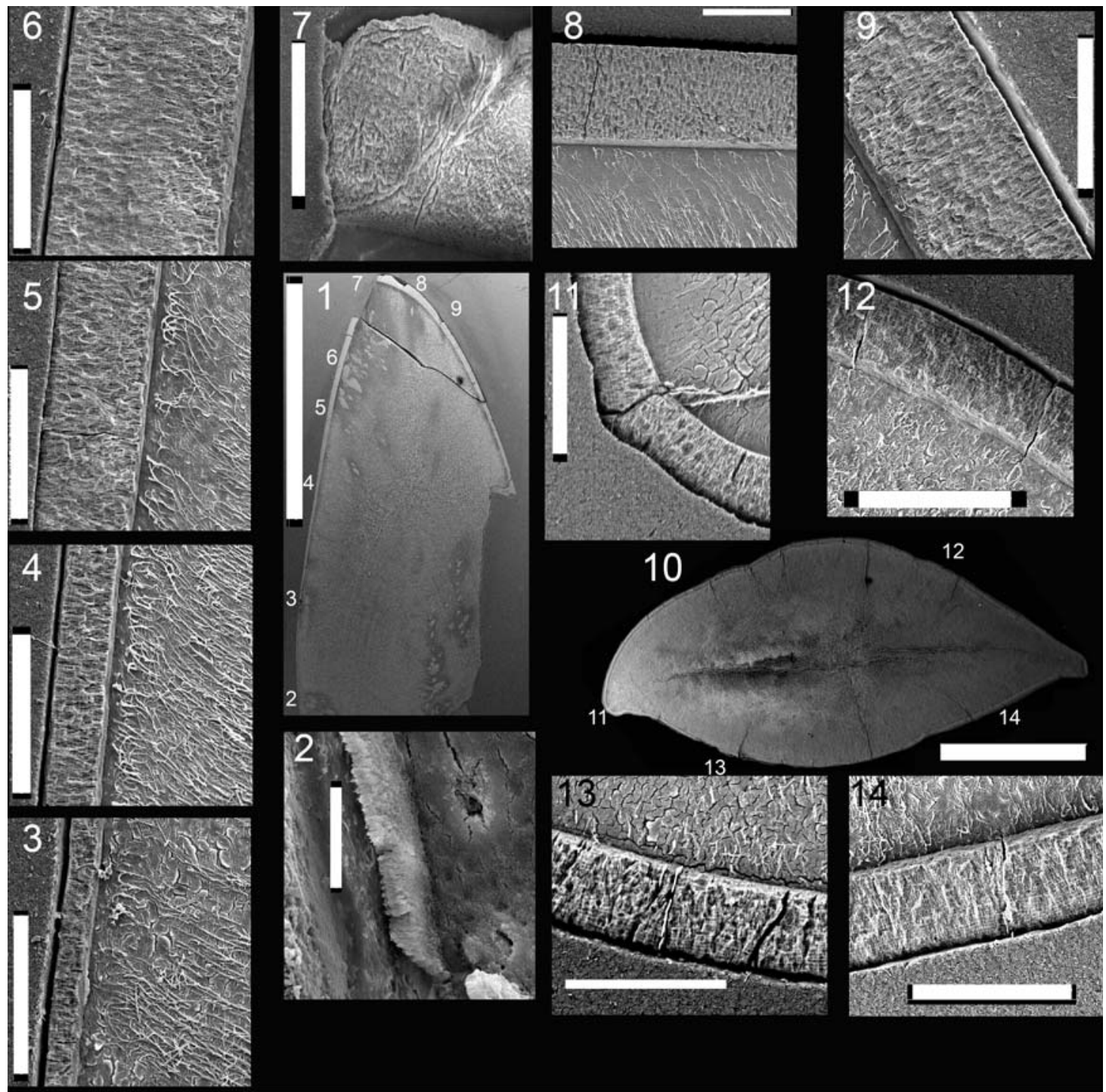

FIGURE 3. Variation in enamel thickness in the teeth of Revueltosaurus callenderi. 1-9, premaxillary tooth (P33799); 10-14, maxillary tooth (P-33798). 1, overview of premaxillary tooth indicating approximate place where measurements and micrographs shown in this figure and Figure 4 were taken; 2-9, close-up views showing enamel thickness variation, with enamel-dentine junction (EDJ) oriented relative to overview in (1); and 10, overview of maxillary tooth section, indicating approximate place where measurements and micrographs shown in this figure and Figure 5 were taken; 11-14, close-up views showing enamel thickness variation, with enamel-dentine junction (EDJ) oriented relative to overview in (10). Scale bars equal $100 \mu \mathrm{m}$ except for 1 (5 mm), 2 (10 $\mu \mathrm{m})$, and 10 (2 mm).

\section{DESCRIPTION}

\section{Revueltosaurus}

Here we provide details of the enamel microstructural features observed in the topotype premaxillary tooth (NMMNH P-33799; Figures 3.1-9, 4) and maxillary-dentary tooth (NMMNH P-33798; Figures 3.10-14, 5) of Revueltosaurus callenderi following the format of Hwang (2005, 2011).

Enamel distribution and thickness. Enamel thickness varied considerably throughout the longi- tudinal section of the premaxillary tooth of Revueltosaurus and very little across the transverse section of the maxillary/dentary tooth (Figures 4, $5)$. In the premaxillary tooth, the enamel was thinnest basally $(\sim 4.9 \mu \mathrm{m})$ and thickest apically (as much as $152 \mu \mathrm{m}$ ), with average enamel thickness approximately $66 \mu \mathrm{m}$ (Table 2), although excluding more basal portions of the tooth (enamel $<40 \mu \mathrm{m}$ thick) results in an average enamel thicknesses of approximately $83 \mu \mathrm{m}$ in the more functional portion of the tooth crown. The enamel in the maxillary- 


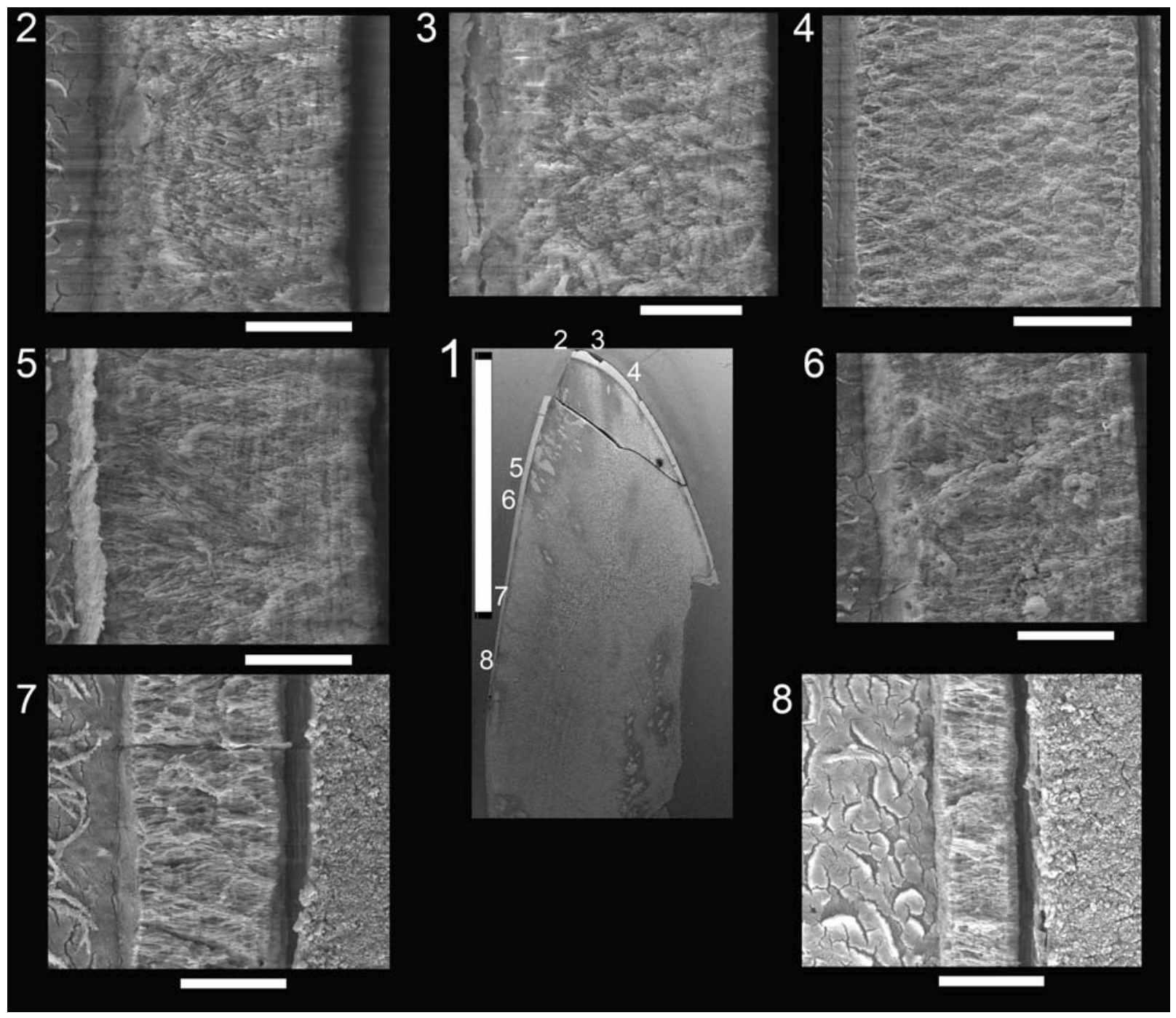

FIGURE 4. Scanning electron microscope images of NMMNH P-33799, Revueltosaurus callenderi premaxillary tooth showing variation in enamel microstructure in longitudinal section. 1, overview of tooth indicating approximate place where measurements and micrographs shown in this figure were taken; 2-8, close-up views showing enamel thickness variation, with enamel-dentine junction (EDJ) oriented relative to overview in (1) and outer enamel surface (OES) away from the same overview. White scale bars equal $20 \mu \mathrm{m}$ except for $1(5 \mathrm{~mm})$ and $4(50 \mu \mathrm{m})$.

dentary tooth was almost uniformly 50-55 $\mu \mathrm{m}$ thick except across the denticles, where it thickened to as much as $92.0 \mu \mathrm{m}$ but was more typically $\sim 60-63$ $\mu \mathrm{m}$. Average transverse thickness was approximately $55.5 \mu \mathrm{m}$ (discounting the one $92.0 \mu \mathrm{m}$ measurement) and $58.5 \mu \mathrm{m}$ counting all measurements, including one thin $(42 \mu \mathrm{m})$ measurement.

Enamel types and schmelzmuster. Typically Revueltosaurus teeth have a thin $(<5 \mu \mathrm{m})$ but welldeveloped BUL from which weakly developed columnar enamel emanates (Figures 4, 5). Individual columnar units are difficult to discern but are $\sim 10 \mu \mathrm{m}$ across basally and expand to $\sim 15-20 \mu \mathrm{m}$ across closer to the OES. Generally these are better seen in transverse sections in the maxillarydentary tooth (Figure 5.2, 5.4-5) than in the premaxillary tooth (Figure 4.2). The outer quarter to half of the enamel thickness bears numerous welldeveloped LIGs. Again, these are more distinct in the transversely sectioned maxillary tooth (Figure 5.2-8) than in the longitudinal section of the premaxillary tooth (Figure 4.2, 5, 8). Rarely LIGs are evident for more than half of the tooth's enamel thickness. LIGs are less than $2 \mu \mathrm{m}$ apart and, in transverse section, approximately 15 can be traced across a micrograph (Figure 5.5-8). They are fainter and difficult to trace, but much more numer- 


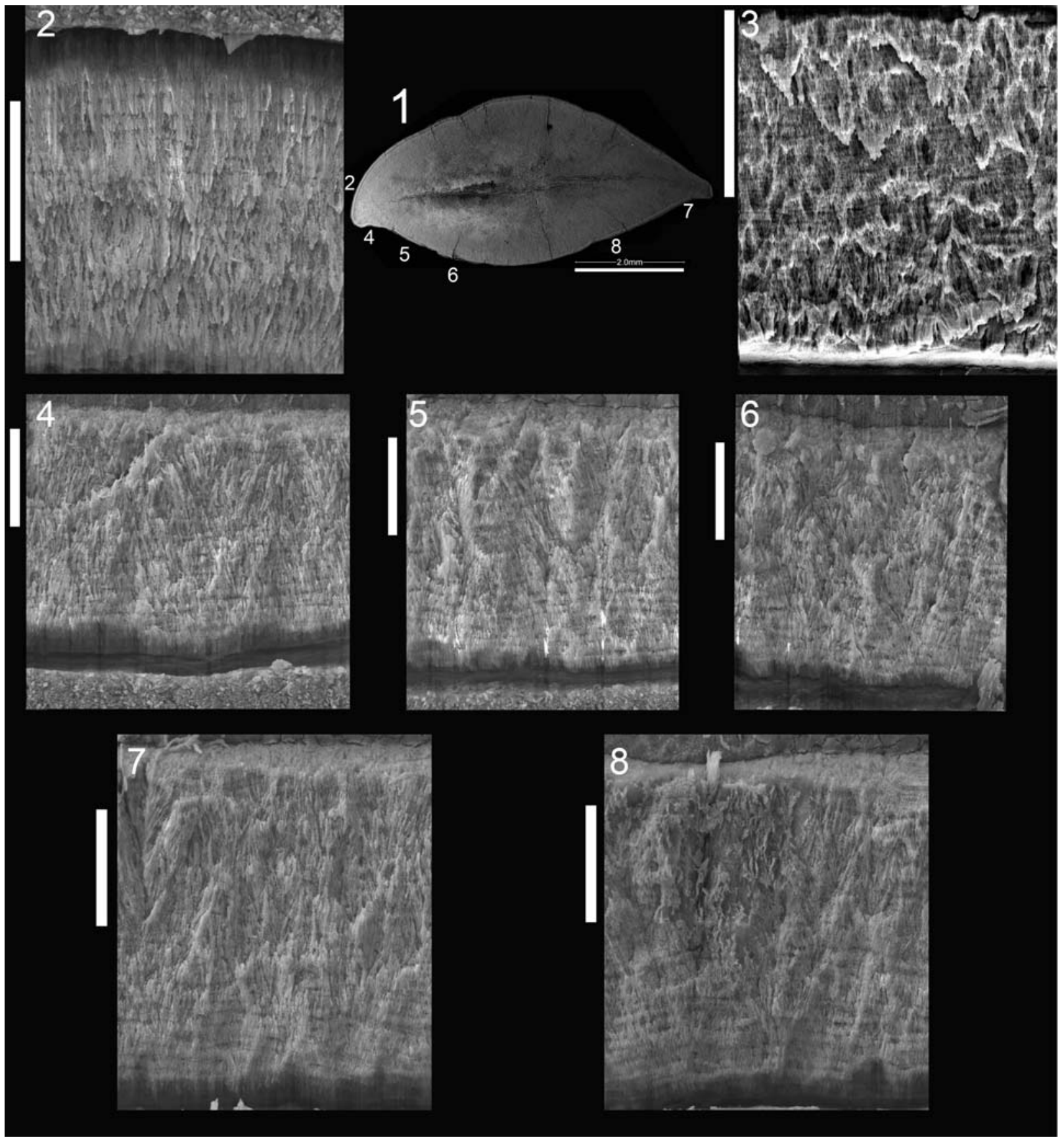

FIGURE 5. Scanning electron microscope images of NMMNH P-33798, Revueltosaurus callenderi maxillary tooth enamel microstructure in transverse section. 1, overview of tooth indicating approximate place where measurements and micrographs shown in this figure were taken; 2-8, close-up views showing enamel thickness variation, with EDJ oriented relative to overview in (1) and OES away from the same overview. Scale bars equal $20 \mu \mathrm{m}$ except 1 (2 mm), $3(50 \mu \mathrm{m})$.

ous in the tip of the premaxillary tooth (Figure 4.4). Near the OES, in the region with the most LIGS, the enamel reverts to a stacked series of parallel crystallites that accounts for approximately $10 \mu \mathrm{m}$ of the total enamel thickness.
Special types and features. The densely packed LIGs are probably the most distinctive feature of the schmelzmuster in Revueltosaurus callenderi. The fact that they are both prominent and densely packed may reflect particularly slow ontogenetic 
Heckert and Miller-Camp: Revueltosaurus tooth enamel

TABLE 2. Measurements of enamel thickness in the teeth of Revueltosaurus and Krzyzanowskisaurus sampled here.

Revueltosaurus callenderi enamel measurements from longitudinal section of NMMNH P-33798

\begin{tabular}{llll} 
Figure & Section & $\begin{array}{c}\text { Enamel thickness } \\
(\boldsymbol{\mu m})\end{array}$ & \multicolumn{1}{c}{ Measured at: } \\
3.2 & Longitudinal & 4.87 & $\begin{array}{l}\text { end of [full width of] enamel (thinnest } \\
\text { enamel) }\end{array}$ \\
3.3 & Longitudinal & 20.42 & $\begin{array}{l}\text { middle of frame } \\
3.4\end{array}$ \\
3.5 & Longitudinal & 36.23 & middle of frame \\
3.6 & Longitudinal & 68.48 & middle of frame \\
3.7 & Longitudinal & 115.56 & top of frame (thickest enamel) \\
3.8 & Longitudinal & 114.88 & left of frame (thickest enamel) \\
3.9 & Longitudinal & 152.13 & bottom of frame (thickest enamel) \\
4.2 & Longitudinal & 103.34 & middle of frame \\
4.3 & Longitudinal & 46.88 & middle of frame \\
4.4 & Longitudinal & 50.08 & middle of frame \\
4.5 & Longitudinal & 52.43 & middle of frame \\
4.6 & Longitudinal & 53.93 & middle of frame \\
4.7 & Longitudinal & 15.47 & middle of frame \\
4.8 & Longitudinal & 31.36 & middle of frame \\
"Tooth has total crown height $(\mathrm{TCH})$ of $13.1 \mathrm{~mm}$, total crown length (TCL) $8.1 \mathrm{~mm} "$
\end{tabular}

\begin{tabular}{|c|c|c|c|}
\hline Figure & Section & $\begin{array}{l}\text { Enamel thickness } \\
\qquad(\mu \mathrm{m})\end{array}$ & Measured at: \\
\hline 3.11 & Transverse & 51.5 & on denticle \\
\hline 3.12 & Transverse & 53.41 & middle of frame \\
\hline 3.13 & Transverse & 55.15 & middle of frame \\
\hline 3.14 & Transverse & 59.66 & middle of frame \\
\hline 5.1 & Transverse & 42.8 & left of frame (EDJ visible) \\
\hline 5.3 & Transverse & 92.47 & middle of frame \\
\hline 5.4 & Transverse & 50.77 & middle of frame \\
\hline 5.5 & Transverse & 54.48 & middle of frame \\
\hline 5.6 & Transverse & 53.32 & middle of frame \\
\hline 5.7 & Transverse & 62.79 & middle of frame \\
\hline 5.8 & Transverse & 60.04 & middle of frame \\
\hline $\begin{array}{l}\mathrm{TCH}: 6 \mathrm{~mm}+ \\
\text { (broken) }\end{array}$ & & $\mathrm{TCL}: \sim 6 \mathrm{~mm}$ & \\
\hline
\end{tabular}

development of each tooth (see text in comparisons and discussion that follows).

\section{Krzyzanowskisaurus}

Here we provide details of the enamel microstructural features observed in the hypothesized premaxillary tooth (UCMP 165213; Figure 6) and the hypothesized maxillary-dentary tooth (UCMP
165211; Figure 7) of Krzyzanowskisaurus hunti following the format of Hwang (2005, 2011).

Enamel distribution and thickness. As in Revueltosaurus, the greatest variation in enamel thickness is through the longitudinal section, which ranged from 18-151 $\mu \mathrm{m}$ thick (Table 2), although only the thicker, more apical sections were preserved well and thus are illustrated in Figure 6. As in Revueltosaurus, Krzyzanowskisaurus had very 
TABLE 2 (continued).

Revueltosaurus callenderi enamel measurements from longitudinal section of NMMNH P-33798

\begin{tabular}{cccl}
$\begin{array}{c}\text { Krzyzanowskisaurus hunti enamel } \\
\text { Figure }\end{array}$ & Section & $\begin{array}{c}\text { measurements from longitudinal section of UCMP } 165213 \\
\text { Enamel thickness } \\
(\mu \mathrm{m})\end{array}$ & \multicolumn{1}{c}{ Measured at: } \\
6.2 & Longitudinal & 112.8 & middle of frame \\
6.3 & Longitudinal & 138.89 & left of frame (EDJ visible) \\
6.4 & Longitudinal & 151.25 & right of frame (unbroken outer edge of \\
& & & enamel) \\
TCH: $\sim 6 \mathrm{~mm}$ & TCL: $6 \mathrm{~mm}$ & \\
(incomplete) & &
\end{tabular}

Krzyzanowskisaurus hunti enamel measurements from transverse sectionof UCMP 165211

$\begin{array}{cccl}\text { Figure } & \text { Section } & \begin{array}{c}\text { Enamel thickness } \\ (\boldsymbol{\mu m})\end{array} & \text { Measured at: } \\ 7.1 & \text { Transverse } & 55.38 & \text { middle of bend } \\ 7.2 & \text { Transverse } & 64.34 & \text { middle of frame } \\ 7.3 & \text { Transverse } & 50.49 & \text { middle of frame } \\ 7.4 & \text { Transverse } & 65.54 & \text { middle of frame } \\ 7.5 & \text { Transverse } & 63.38 & \text { middle of frame } \\ 7.6 & \text { Transverse } & 43.47 & \text { middle of frame } \\ 7.7 & \text { Transverse } & 61.82 & \text { middle of frame } \\ \text { TCH: } \sim 10.4 \mathrm{~mm} & \text { TCL: } \sim 7.4 \mathrm{~mm} & & \end{array}$

thin enamel basally and the thickest enamel apically. All of the reasonably well-preserved, more apical sections illustrated in Figure 6 have enamel more than $100 \mu \mathrm{m}$ thick. The complex shape of the transverse section (see Figure 7) led to samples of very thin enamel between the denticles and main body of the tooth, and much thicker enamel across the denticles more mesially and distally and thus ranged from 43.5 to $65.5 \mu \mathrm{m}$ thick. Typical thicknesses were $\sim 50-65 \mu \mathrm{m}$ with an average thickness of approximately $58 \mu \mathrm{m}$.

Enamel types and schmelzmuster. The EDJ is clearly demarcated in both the longitudinal and transverse section and marked with a distinct BUL of small, diffuse columns. This BUL is also less than $5 \mu \mathrm{m}$ thick. In both longitudinal and transverse sections the individual columns are relatively small and defined by packages of crystallites $\sim 5 \mu \mathrm{m}$ across and $10 \mu \mathrm{m}$ long. In some views (e.g., Figure $6.3-5,7.3,7.5,7.7)$ it appears that the enamel is comprised of at least two, and sometimes three or four, "stacks" of such columns. LIGs are particularly visible in transverse section but much less than 2 $\mu \mathrm{m}$ apart, so as many as 25 are evident in some teeth (Figure 7.3, 7.6). As in Revueltosaurus, there is a transtion within the LIGS from the more basal poorly developed columnar units to parallel crystallite enamel, which dominates the outermost $\sim 10$ $\mu \mathrm{m}$ of the tooth (Figures 6.2, 7.3-7).

Special types and features. In many ways the schmelzmuster of Krzyzanowskisaurus hunti is extremely similar to that of Revueltosaurus callenderi, from its thickness to the presence of numerous LIGs. The teeth sampled for this study were approximately the same size, and the enamel thickness in each taxon is essentially the same. Similarly, the schmelzmuster of $K$. hunti, as in $R$. callenderi, consists of a BUL from which columnar enamel emanates, bears numerous closely spaced LIGs that are much more prominent near the OES than near the enamel-dentine junction, and grades into parallel crystallite enamel near the OES. The significance of these features and their similarities are discussed in the following sections.

\section{COMPARISONS}

Both Revueltosaurus and Krzyzanowskisaurus have relatively thick enamel for teeth of their size-enamel thickness varies from a low of $\sim 5 \mu \mathrm{m}$ 


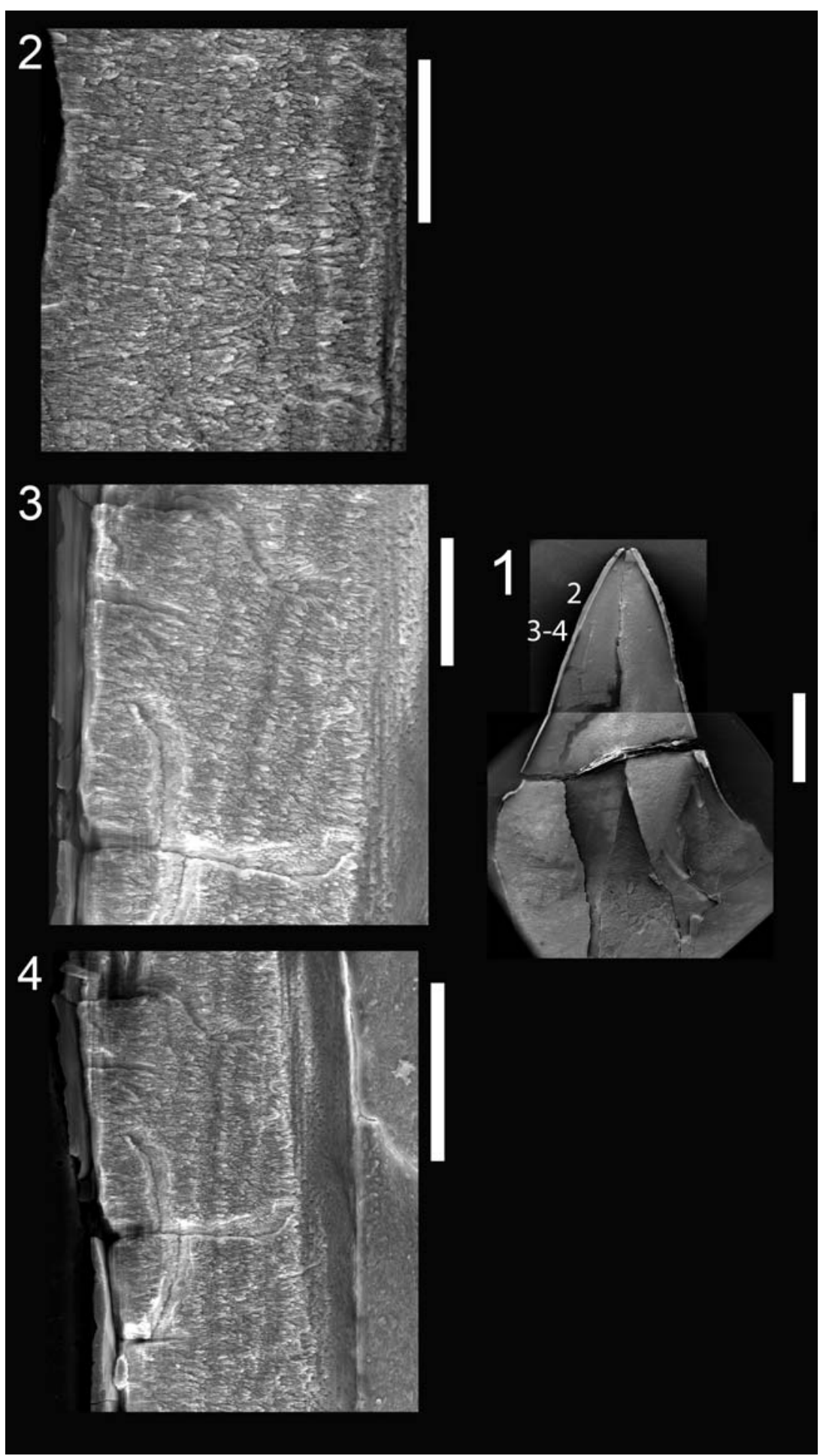

FIGURE 6. Scanning electron microscope images of UCMP 165213, Krzyzanowskisaurus hunti tooth enamel microstructure in longitudinal section. 1, overview of tooth indicating approximate place where measurements and micrographs shown in this figure were taken; 2-4, close-up views showing enamel thickness variation, with EDJ oriented relative to overview in (1) and OES away from the same overview. Scale bars equal $2 \mathrm{~mm}(1), 50 \mu \mathrm{m}(2-3)$, and 100 $\mu \mathrm{m}$ (4). 


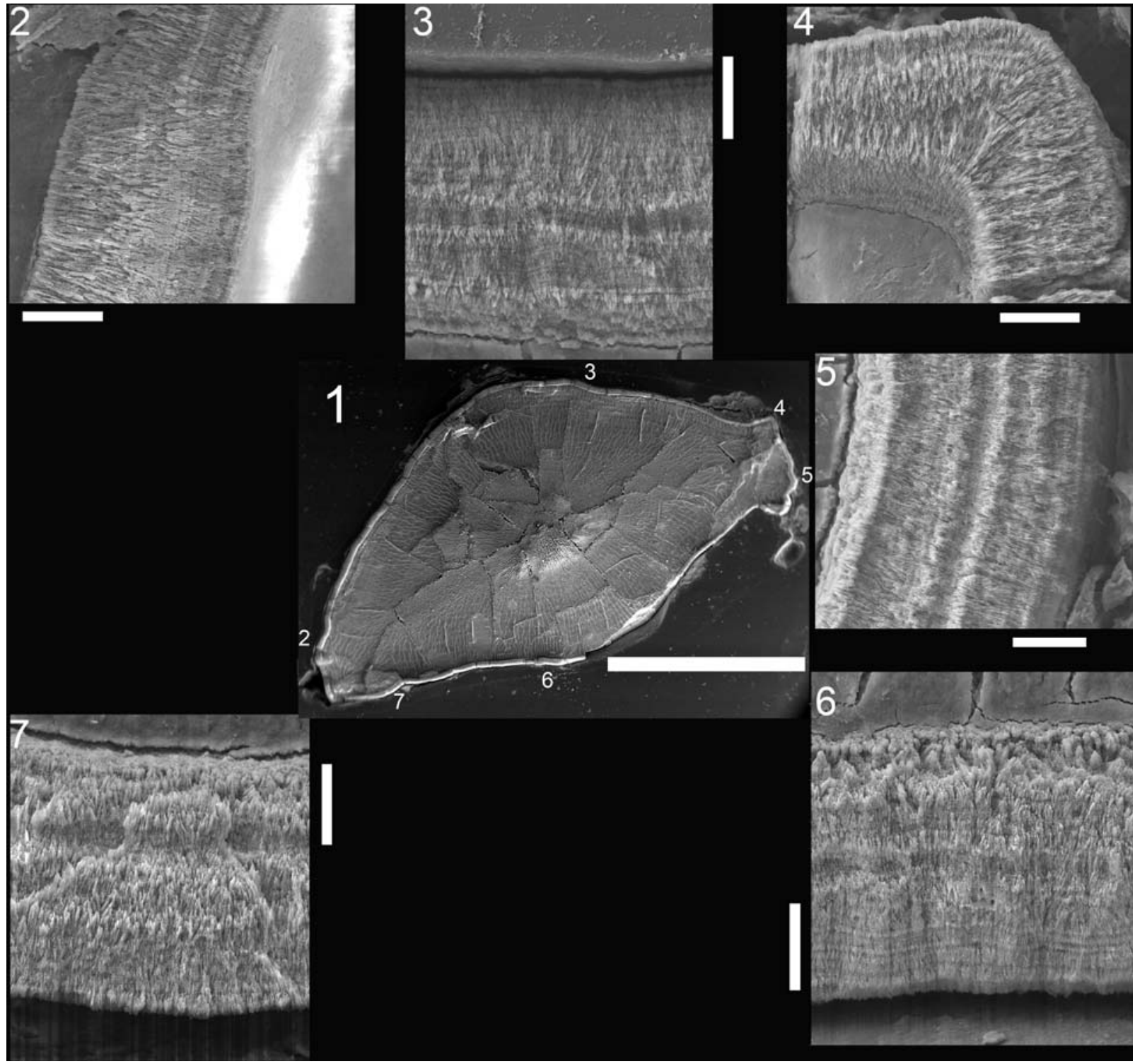

FIGURE 7. Scanning electron microscope images of UCMP 165211, Krzyzanowskisaurus hunti premaxillary tooth enamel microstructure in transverse section. 1, overview of tooth indicating approximate place where measurements and micrographs shown in this figure were taken; 2-7, close-up views showing enamel thickness variation, with EDJ oriented relative to overview in (1) and OES away from the same overview. Scale bar equals $20 \mu \mathrm{m}$ except 1 (2 mm).

to as much as $152 \mu \mathrm{m}$. Even though the "premaxillary" tooth of Krzyzanowskisaurus is approximately $25 \%$ shorter, the enamel is comparable in thickness. The longitudinal sections of the premaxillary teeth capture the entire range of variation from more basal enamel $(\sim 5 \mu \mathrm{m})$ to apical enamel $(\sim 152$ $\mu \mathrm{m})$. In the maxillary/dentary teeth, the range was not as pronounced (generally 50-65 $\mu \mathrm{m}$, with one measurement each above and below this range), which probably reflects relatively uniform thickness in cross-section, although the enamel is thicker along the denticles. At the microstructural level, both taxa exhibit columnar microstructure that emanates from a BUL. The columnar enamel is then interrupted by numerous LIGS before transitioning into a region $\sim 10 \mu \mathrm{m}$ thick dominated by parallel (crystallite) enamel that extends to the OES. In amniotes, parallel (crystallite) enamel is generally considered primitive and columnar enamel derived, with the assumption that columnar enamel is perhaps associated with a diet requiring greater tooth strength (resistance to fracture) (Sander, 1999). Existing theories suggest that columnar enamel in these taxa reflects a diet with 
less emphasis on grinding and more on biting (Sander, 1999), something we explore in greater detail later.

Of the many taxa Sander (1999) sampled, Revueltosaurus teeth are most similar in enamel thickness and microstructure to rauisuchids (Sander, 1999, plate 9f) among contemporaneous taxa, and tyrannosaurs (Sander, 1999, plate 13bc) among more derived, younger taxa. The similarities with the rauisuchid tooth include the presence of a BUL, weakly developed columnar enamel, and numerous LIGs. The similarities with the rauisuchids taxa include the presence of a BUL, weakly developed columnar enamel, and numerous LIGs, although the enamel of the rauisuchid illustrated by Sander (1999, plate 9f) appears to average almost $100 \mu \mathrm{m}$ thick. Certainly, some phytosaurs (e.g., Sander, 1999; Figure 6a) exhibit better-developed columns, something we have seen in our own preliminary work (Camp and Heckert, 2007). Interestingly, the enamel of both Revueltosaurus and Krzyzanowskisaurus is substantially thicker than that of Trilophosaurus buettneri (e.g., Sander, 1999, plate 9d-e), one of the few other possibly herbivorous tetrapods known from the Upper Triassic of the American Southwest. The columns we illustrate here are better developed than those of Plateosaurus (Sander, 1999, plate $13 \mathrm{~h}$ ) but not as well developed as in the tyrannosaurid illustrated by Sander (1999, plate 13b-c). As in Diplodocus (Sander, 1999, plate 14a-b), the columnar units are more readily discerned in crosssection than longitudinal section. The enamel microstructure of Revueltosaurus callenderi is also similar to that of the ankylosaurid "Palaeoscincus" (Sander, 1999, plate 14f). Sander (1999) documented several sauropsid taxa that possessed both parallel crystallite and columnar enamel, including some ichthyosaurs and mosasaurs, but the schmelzmuster we document here is more homogenous than Sander found in those taxa.

Stokosa (2005; see also Stokosa, 2001) made extensive comparisons of small (Troodon, Paronychodon, Richardoestesia, Dromaeosaurus) and large (tyrannosaurid) latest Cretaceous theropods. Generally speaking, she found that the smaller taxa, particularly Troodon and Richardoestesia, lack columnar enamel and other more complex structural features, and thus are very different from the schmelzmuster we document in Revueltosaurus and Krzyzanowskisaurus. Although Stokosa's (2005) analysis was restricted to Late Cretaceous coelurosaurian theropods, we note that she also reported columnar enamel similar to what we report here in several dromaeosaurid and tyrannosaurid theropods (but note that Hwang [2011] considered Stokosa's [2005] "columnar" enamel as a BUL). The columns we document here are not as well developed as those illustrated by Stokosa (2005, figure 9.6c-d) for Tyrannosaurus rex. Conversely, the LIGs we report here are more pronounced than those Stokosa (2005, figure 9.3c) found in Dromaeosaurus.

Hwang's (2005, 2009, 2011) work was restricted to the Dinosauria but is the most comprehensive sampling of dinosaurs to date. Again, enamel thickness in Revueltosaurus and Krzyzanowskisaurus is comparable to that found in much larger teeth (e.g., the tyrannosaurid theropods, Table 1). Other key features we observed in the schmelzmuster of Revueltosaurus and Krzyzanowskisaurus include a BUL, columnar enamel consisting of relatively small $(<15 \mu \mathrm{m}$ diameter) columns, and numerous LIGs that mark a transition from columnar to parallel crystallite enamel. Hwang (2011, figure 18) reported an identical combination of features in the four genera of ankylosaurs she sampled. Indeed, the schmelzmuster of the Triassic taxa we sampled is extremely similar to that Ankylosaurus magniventris as illustrated by Hwang (2005, figure $10 \mathrm{a}-\mathrm{c}$; Hwang, 2011, figure 13a). Points of detailed similarity include the comparable enamel thickness, thin but well-defined BUL, and poorly developed columns basally that grade into a zone with pronounced LIGS before transitioning into a zone of parallel crystallite enamel (see especially Hwang, 2005 , figure $10 a, c)$. A single unidentified pachycephalosaurid also possessed similar enamel, although it has what Hwang (2011) identified as "incipient columnar enamel" rather than the true columnar enamel identified here. The hypsilophodont ornithischian Thescelosaurus possesses similar schmelzmuster, including a BUL, columns, and at least some LIGs (accidentally omitted from Hwang, 2011, figure 18), but also possesses a parallel crystallite enamel cap forming the outer enamel surface similar to that of the thyreophorans. The presence of a BUL and relatively narrow columns is probably synapomorphic for ornithischians (Hwang, 2011), but no ornithischian exactly matches the schmelzmuster characteristics of either Revueltosaurus or Krzyzanowskisaurus. We therefore consider these similarities to be the result of convergence and find it intriguing that this convergence appears strongest with ankylosaurs, whose teeth are also superficially similar to the Tri- 
assic taxa, especially those of Krzyzanowskisaurus.

Several theropods and a few of the sauropodomorphs Hwang $(2005,2011)$ sampled share one or two features (BUL, narrow columnar enamel, LIGs) with Revueltosaurus and Krzyzanowskisaurus, but none share all three, and in general the schmelzmuster of saurischians is distinct from these taxa. Interestingly, Triassic saurischians, including both Coelophysis and the "prosauropods" (basal sauropodomorphs) Plateosaurus and "Gyposaurus" have less sophisticated tooth enamel microstructure than do the taxa examined here (Sander, 1999; Hwang, 2005, 2011).

\section{DISCUSSION}

These results demonstrate that (1) enamel thickness is variable within a tooth; therefore, enamel thickness reports must either be standardized to a specific location on a tooth (which we consider unlikely to be repeatable in archosaurs) or else reported as a range tied to morphological landmarks (which we think more likely and have practiced here); (2) Revueltosaurus and Krzyzanowskisaurus both had columnar enamel microstructure through most of the enamel, but grading into an outer portion composed of parallel crystallite enamel, suggesting selection for more durable tooth enamel; (3) the numerous LIGS in these teeth suggest that they may have had a lengthy developmental stage and were retained in the jaw for long periods of time; and (4) the overall similarity of microstructural features (schmelzmuster) in Krzyzanowskisaurus and Revueltosaurus lends credence to the hypothesis that the two taxa are in fact closely related.

Regarding the first point, most, if not all, workers have acknowledged the variable thickness of reptilian tooth enamel. However, what is lacking in most reports, even the relatively comprehensive approaches of Sander (1999) and Hwang (2005), is any standardized assessment of this variation (note the many "holes" in Table 1, especially with regard to minimal enamel thickness). This has been addressed somewhat in more recent papers (Hwang, 2009, 2011), but there is still a need for standardization, especially given the relative ease of image acquisition and digital measurement now compared to the first tooth enamel microstucture workers. Sources of variation evident in this study include tooth position, location on the tooth, and, potentially, taxonomic position. Future studies need to better document tooth size, shape, and when possible, position prior to embedding and sectioning, so that sources of thickness variation are better constrained and therefore understood. We feel that there is real need to compare actual tooth size to enamel thickness, but with the exception of Hwang's most recent (2011) paper, whole tooth measurements are lacking in the literature. As an example of how these comparisons might be significant, consider that the maximum enamel thickness we report here for Revueltosaurus is similar to that reported in many tyrannosaurs (Sander, 1999; Hwang, 2005; Stokosa, 2005). However, the teeth we sectioned are more than an order of magnitude smaller in total crown height and significantly smaller in crown width and length than tyrannosaur teeth, and thus are probably, volumetrically, nearly two orders of magnitude smaller than tyrannosaur teeth. Almost surely there is some biological significance to the fact that, in spite of this volumetric difference, Revueltosaurus and Krzyzanowskisaurus had enamel nearly as thick as that of Tyrannosaurus, but without more rigorous, standardized reporting protocols, this fact is easily lost. There is, as Sander (1999) and Hwang (2005, 2011) admitted, certainly much homoplasy among tooth enamel microstructure across sauropsids. One fact to consider in this analysis is that Revueltosaurus and Krzyzanowskisaurus teeth are similar in size to those of theropods much smaller than $T$. rex, and that tyrannosaurs doubtless possessed some of the largest teeth of any reptile, although Hwang (2005) noted that tyrannosaurid teeth possess proportionately thin enamel relative to their size. The underlying biological reasons for this variation can only be investigated if the variation itself is documented, as we strive do to here.

The relatively thick enamel of Revueltosaurus and Krzyzanowskisaurus, especially given the small size of the teeth, suggests selection for teeth particularly resistant to abrasion. Although much thinner than the enamel of some of the durophagous taxa examined by Sander (1999), Revueltosaurus and Krzyzanowskisaurus teeth have enamel that is thicker than that of most relatively closely related taxa, especially when controlled for size. With their phyllodont (leaf-like) to spatulate shape and relatively coarse, non-perpendicular denticles, the teeth of both taxa fit within the broadly defined "non-oral processing herbivore" adaptive complex of Sander (1999), and possess many characteristics considered "putatively herbivorous traits" by Zanno and Mackovicky (2011), who focused on dinosaurs but still produced hypotheses relevant to more basal archosauriformes. How- 
ever, tooth enamel microstructure provides few additional clues to the diet of either taxon. Sander (1999) noted that columnar enamel appears lesswell adapted to true grinding surfaces than does parallel crystallite enamel, based in part on the widespread occurrence of thin caps of parallel crystallite enamel atop the thick columnar enamel of many undoubtedly durophagous reptiles. On the other hand, Sander (1999) and Hwang (2005, 2007, 2011) have documented schmelzmuster of almost entirely columnar enamel in many taxa whose teeth were clearly involved in grinding, such as ceratopsian dinosaurs. However, these taxa actively abrade the tooth through the outer enamel surface, so this may be different than the parallel crystallite enamel "cap" of durophagous taxa. Columnar enamel is thought to better resist bending forces, especially in reptiles where the enamel tends to be thin relative to the dentine, and must therefore accommodate not only external stresses, but also the resulting strain on the dentine (e.g., Koenigswald and Pfretzschner, 1992; Rensberger, 1997; Sander, 1999). Sander (1999) thus considered parallel crystallite enamel superior to columnar in terms of resisting wear and abrasion, but columnar enamel superior to parallel crystallite enamel in resisting cracking and bending. The presence of parallel crystallite enamel in the outer portions of the teeth in Revueltosaurus and Krzyzanowskisaurus is thus somewhat similar to durophagous taxa documented by Sander (1999), although the Triassic enamel is much thinner (< $100 \mu \mathrm{m})$ than some of the mosasaur, crocodilian, and ichthyosaur specimens examined by Sander (1999). It thus appears likely that both Revueltosaurus and Krzyzanowskisaurus ate items that placed a greater emphasis on biting than on true grinding, even though Heckert (2002, 2005) demonstrated relatively precise abrasion of the denticles in both taxa. At present it is not possible to accurately discern the shape of the skull of Revueltosaurus (e.g., Parker et al., 2005), so it is unclear whether this wear might be the result of feeding habits, such as ingesting or stripping twigs and branches or other roughage while browsing or eating Triassic ground cover or roots and other subterranean plant matter mixed with soil while "grazing."

Both Revueltosaurus and Krzyzanowskisaurus possess numerous LIGs. These are especially apparent in the outer layers of the Revueltosaurus maxillary-dentary tooth in transverse section, but remain apparent even in the thin enamel near the base of the premaxillary tooth. Revueltosaurus
LIGs are both well developed and numerous (20+ bands apparent in Figure 4.4), suggesting a lengthy growth period for each tooth. Indeed, these LIGs are similar in terms of both frequency and dominance of the enamel microstructure to that of a rauisuchian illustrated by Sander (1999, plate 9f). Generally speaking, LIGs are more prevalent in taxa with parallel crystallite than columnar enamel, but do occur in many of the taxa that bracket both Revueltosaurus and Krzyzanowskisaurus phylogenetically. However, the frequency of LIGs seen in the teeth here far outstrips that of any comparably sized taxon in Sander (1999) or Hwang (2011), suggesting that these teeth were slower-developing and/or retained longer than in, for example, ornithischian dinosaurs. Ornithischians with relatively similar patterns of LIGs include an indeterminate ankylosaur and Thescelosaurus (Sander, 1999, plate $14 \mathrm{~g}-\mathrm{h}$, respectively), although we note that Hwang (2011, figure 16a-c) reported a different schmelzmuster in Thescelosaurus, casting some doubt on the affinities of the Thescelosaurus tooth illustrated by Sander (plate 14h). There are examples of presumably fast-growing taxa, such as troodontid theropods, with numerous LIGS (Hwang, 2005, 2011), and the relatively slow-growing extant Uromastyx is the only reptile known to have "mammalian-like" prismatic enamel (Sander, 2000). Appenzeller et al. (2005) posited that LIGS (striae of Retzius) are actually tied to diurnal rhythms of the autonomous nervous system. If this is correct, then the developed ment of these teeth was probably on the order of two weeks to a month, assuming no remodeling. What is intriguing about the Appenzeller et al. (2005) study is that these striae are found across a wide range of taxa, yet are absent from an equally wide assortment of taxa as well.

Teeth assigned to Krzyzanowskisaurus hunti by Heckert (2005) had variously been considered conspecific (Long and Murry, 1995) or congeneric (Heckert, 2002) with Revueltosaurus callenderi based on overall similarity. Parker et al. (2005) and later Irmis et al. (2007; see also Nesbitt et al., 2007) argued that, while distinct at the specific level, $K$. hunti teeth should be assigned to Revueltosaurus. Heckert's (2005) designation of a new species was in part an effort to demonstrate the more ornithischian-like characteristics of $K$. hunti. Parker et al. (2005) and Irmis et al. (2007) in turn argued, in part, that Revueltosaurus osteoderms and an isolated squamosal from the same locality (UCMP 7308) in the UCMP collections supported their taxonomic argument. As Heckert (2005) 
noted, this argument is weakened by the disarticulated nature of material from UCMP 7308, which yields an extensive, and largely undescribed, fauna ranging from osteichthyans to phytosaurs and aetosaurs (see also Long and Murry, 1995; Heckert et al., 2005).

One point of this study was to see if tooth enamel microstructure could provide additional insight into the relatedness of Revueltosaurus and Krzyzanowskisaurus. Although Sander (1999) reported relatively little phylogenetic signal in tooth enamel microstructural features, Hwang (2005, 2011) has reported a variety of synapomorphies that unite at least some dinosaurian taxa and has argued (Hwang, 2009) that enamel microstructure can even be used to help identify isolated and/or fragmentary teeth.

It is clear from this study that numerous features of the enamel microstructure of $R$. callenderi and $K$. hunti are quite similar. Although only a few other Triassic archosaurs have been sampled, the schmelzmuster of these taxa, with a thin BUL, relatively thick, columnar enamel, and numerous fine LIG are unlike any other taxon reported by Sander (1999) or Hwang (2005, 2011). As part of another project we have begun examining the microstructure of phytosaur teeth, greatly expanding upon Sander's (1999) sample of three teeth, and have not found any teeth with this same combination of features (e.g., Camp and Heckert, 2007). Thus, we are inclined to tentatively accept the hypothesis of Parker et al. (2005; Irmis et al., 2007) that Krzyzanowskisaurus hunti is in fact closely allied to $R$. callenderi. However, we prefer to keep the separate generic name of $K$. hunti, noting that it remains likely that, when more complete Krzyzanowskisaurus fossils are found, they will likely be considered distinct at the generic level. Indeed, throughout the Upper Triassic, archosauriform taxa with distinctive dentitions are almost always assigned to different genera, including both shuvosaurids (Shuvosaurus, Effigia, Nesbitt, 2007) and silesaurids (Silesaurus, Asilisaurus, and Diodorus, Nesbitt et al., 2010; Kammerer et al., 2012). Thus, until more diagnostic skeletal material unambiguously associated with Krzyzanowskisaurus is found, we advocate retaining the two taxa as separate, but closely related genera. Because Revueltosaurus is now widely considered the sister taxon to aetosaurs (e.g., Nesbitt, 2011; Butler et al., 2011), obtaining enamel microstructural details of aetosaurs is highly desirable to see if these complex features (thick, complex enamel with many LIGs, for example) are present in aetosaurs, which have comparatively simple dentitions, albeit with some taxonomic variation (e.g., Walker, 1961; Heckert and Lucas, 2000).

\section{CONCLUSIONS}

Tooth enamel microstructure in Revueltosaurus callenderi and Krzyzanowskisaurus hunti is relatively unique among archosaurs, and the enamel itself is particularly thick for teeth of their size. Neither result is terribly surprising, as Hwang (2011) notes that, in dinosaurs, schmelzmuster complexity correlates with tooth complexity, and these teeth have long been recognized as among the most complex of Triassic archosauriforms. The thickness of the enamel in these teeth appears impressive for their size, although comparisons across taxa using the literature are hampered by a dearth of data reporting original tooth size. The numerous LIGs may hint at teeth with an exceptionally long life in the jaw. Whether this is a reflection of functional utility (teeth well adapted for their function and only rarely shed) or simply an indication of slow growth cannot be determined. The details of the tooth enamel microstructure of both taxa most closely resembles that of ankylosaurs, indicating convergent evolution between the Triassic taxa and the much later ornithischians.

Future studies of archosauriform tooth enamel microstructure should address several problems: (1) Standardization of thickness measurements to landmarks if possible; (2) consideration of enamel thickness and complexity relative to overall tooth size; (3) wider taxonomic sampling, especially among Triassic archosauriforms to determine the extent that these features reveal aspects of the diversification of archosaurs during the Triassic. This is especially important as revueltosaurs, silesaurids, and shuvosaurids, among others, represent early "experiments" in herbivory, or at least are not obviously carnivorous forms, within Archosauria. With more complex methodologies now being employed to analyze dietary preferences in similar taxa, such as Jurassic-Cretaceous theropods (Zanno and Mackovicky, 2011), it is important to establish repeatable, if not quantifiable, descriptions of enamel microstructure characteristics, as these are clearly of great adaptive significance.

\section{ACKNOWLEDGEMENTS}

P. Holroyd (UCMP) and S. Lucas (NMMNH) generously allowed us to borrow and section specimens in their care. Sections of NMMNH specimens were prepared at the University of Bonn 
courtesy of P.M. Sander. A. Love and A. Abernethy assisted in section preparation of UCMP specimens at Appalachian State University (ASU). The College of Arts and Sciences at ASU supported this work by providing nearly unlimited access to the SEM. Drs. G. Hou and S. Hageman helped enormously in this respect. A. Bartholomew-Staples prepared the web versions of Figures 3-7. Numerous discussions with S. Hwang as well as P.M. Sander were extremely helpful, as were unpublished illustrations Hwang generously shared with us. The Department of Geology and the Office of Student Research at ASU supported our efforts to present preliminary results of this research, as did a research poster prize (to JAC) from the Appalachian Regional Electron Microscopy Society. Reviews by P.M. Sander and an anonymous reviewer as well as comments by the Sylvain Gerber and the editorial staff of Palaeontologia Electronica improved the manuscript.

\section{REFERENCES}

Appenzeller, O., Gunga, H.-C., Qualls, C., Furlan, R., Porta, A., Lucas, S.G., Heckert, A.B., Kirsch, K., Costa-Junqueira, M.A., Guillén, S.E., Sander, M., Schneider, T., and Blottner, B. 2005. A hypothesis: autonomic rhythms are reflected in growth lines of teeth in humans and extinct archosaurs. Autonomic Neuroscience: Basic and Clinical, 117:115-119.

Buffetaut, E., Dauphin, Y., Jaeger, J.E., Martin, M., Mazin, J.-M., and Tong, H. 1986. Prismatic dental enamel in theropod dinosaurs. Naturwissenschaften, 73:326-327.

Butler, R.J., Brusatte, S.L., Reich, M., Nesbitt, S.J., Schoch, R.R., and Hornung, J.J. 2011. The sailbacked reptile Ctenosauriscus from the latest Early Triassic of Germany and the timing and biogeography of the early archosaur radiation. PLOS One, 6:e25693.

Camp, J.A. and Heckert, A.B. 2007. Tooth enamel microstructure in Phytosauria (Reptilia: Archosauria) and its implications for phylogeny, ecology, and biostratigraphy. Geological Society of America Abstracts with Programs, 39(2):86.

Dauphin, Y. 1988. L'email dentaire des reptiles actuels et fossiles: Repartition de la structure prismatique, son role, ses implications. Palaeontographica Abteilung A., 203:171-184.

Dauphin, Y., Jaeger, J.-J., and Osmólska, H. 1988a. Enamel microstructure of ceratopsian teeth (Reptilia, Archosauria). Geobios, 21:319-327.

Dauphin, Y., Jaeger, J.-J., and Osmólska, H. 1988b. Microstructure et composition chimique élémentaire des dents de deux dinosaures théropodes du Crétacé supérieur de Mongolie: Velociraptor et Tyrannosaurus (Reptilia, Archosauria). Annales de Paléontologie (Vert-Invert), 75(2):83-98.
Heckert, A.B. 2002. A revision of the Upper Triassic ornithischian dinosaur Revueltosaurus, with a description of a new species. New Mexico Museum of Natural History and Science Bulletin, 21:253-268.

Heckert, A.B. 2005. Krzyzanowskisaurus, a new name for a probable ornithischian dinosaur from the Upper Triassic Chinle Group, Arizona and New Mexico. New Mexico Museum of Natural History and Science Bulletin, 29:77-83.

Heckert, A.B. and Camp, J.A. 2006. Enamel microstructure in Revueltosaurus callenderi (Archosauria: Crurotarsi) an unusual reptile from the Triassic of the American Southwest. Museum of Northern Arizona Bulletin, 62:153-154.

Heckert, A.B. and Lucas, S.G. 1997. First use of ornithischian dinosaurs for biostratigraphic zonation of the Upper Triassic. Albertiana, 20:58-63.

Heckert, A.B. and Lucas, S.G. 2000. Taxonomy, phylogeny, biostratigraphy, biochronology, paleobiogeography, and evolution of the Late Triassic Aetosauria (Archosauria:Crurotarsi). Zentralblatt für Geologie und Paläontologie Teil I 1998 11-12:1539-1587.

Heckert, A.B., Lucas, S.G., and Hunt, A.P. 2005. Triassic vertebrate fossils in Arizona. New Mexico Museum of Natural History and Science Bulletin, 29:16-44.

Hunt, A.P. 1988. The oldest prosauropod dinosaur in North America, from the upper shale member of the Chinle Formation (Late Triassic) in east-central New Mexico. New Mexico Geology, 10:65.

Hunt, A.P. 1989. A new ?ornithischian dinosaur from the Bull Canyon Formation (Upper Triassic) of east-central New Mexico, p. 355-358. In Lucas, S.G. and Hunt, A.P. (eds.), Dawn of the age of dinosaurs in the American Southwest. New Mexico Museum of Natural History, Albuquerque.

Hunt, A.P. and Lucas, S.G. 1994. Ornithischian dinosaurs from the Upper Triassic of the United States, p. 227-241. In Fraser, N.C. and Sues, H.-D. (eds.), In the shadow of the dinosaurs: Early Mesozoic tetrapods. Cambridge University Press, Cambridge.

Hunt, A.P., Lucas, S.G., and Spielmann, J.A. 2005. The postcranial skeleton of Revueltosaurus callenderi (Archosauria: Crurotarsi) from the Upper Triassic of Arizona and New Mexico, USA. New Mexico Museum of Natural History and Science Bulletin, 29:67-76.

Hunt, A.P., Lucas, S.G., Heckert, A.B., Sullivan, R.M., and Lockley, M.G. 1998. Late Triassic dinosaurs from the western United States. Geobios, 31:511-531.

Hwang, S.H. 2005. Phylogenetic patterns of enamel microstructure in dinosaur teeth. Journal of Morphology, 266:208-240.

Hwang, S.H. 2006. Phylogenetic analysis of enamel microstruture characters in dinosaur teeth. Journal of Vertebrate Paleontology, 26(Supplement to no. 3):80A.

Hwang, S.H. 2007. Phylogenetic patterns of enamel microstructure in dinosaur teeth. Unpublished PhD Thesis, Columbia University, New York, USA. 
Hwang, S.H. 2009. The utility of tooth enamel microstruture in identifying isolated dinosaur teeth. Lethaia:116.

Hwang, S.H. 2011. The evolution of dinosaur tooth enamel microstructure. Biological Reviews, 86:183216.

Irmis, R.B., Parker, W.G., Nesbitt, S.J., and Liu, J. 2007. Early ornithischian dinosaurs: the Triassic record. Historical Biology, 19:3-22.

Kammerer, C.F., Nesbitt, S.J., and Shubin, N.H. 2012. The first silesaurid dinosauriform from the Late Triassic of Morocco. Acta Palaeontologica Polonica, 57:277-284.

Koenigswald, W.v. and Pfretzchner, H.-U. 1992. Biomechanics in the enamel of mammalian teeth, p. 113125. In Schmidt-Kittler, N. and Vogel, K. (eds.), Constructional morphology and evolution. Springer-Verlag, Berlin.

Koenigswald, W.v. and Sander, P.M. (eds) 1997. Tooth enamel microstructure. A.A. Balkema, Rotterdam.

Long, R.A. and Murry, P.A. 1995. Late Triassic (Carnian and Norian) tetrapods from the southwestern United States. New Mexico Museum of Natural History and Science Bulletin, 4:1-254.

Lucas, S.G., Hunt, A.P., Heckert, A.B., and Spielmann, J.A. 2007. Global Triassic tetrapod biostratigraphy and biochronology: 2007 status. New Mexico Museum of Natural History and Science Bulletin, 41:229-240.

Nesbitt, S.J. 2007. The anatomy of Effigia okeeffeae (Archosauria, Suchia), theropod-like convergence, and the distribution of related taxa. Bulletin of the American Museum of Natural History, 302:84.

Nesbitt, S.J. 2011. The early evolution of archosaurs: relationships and the origin of major clades. Bulletin of the American Museum of Natural History, 392:1292.

Nesbitt, S.J., Irmis, R.B., and Parker, W.G. 2007. A critical re-evaluation of the Late Triassic dinosaur taxa of North America. Journal of Systematic Palaeontology, 5:209-243.

Nesbitt, S.J., Sidor, C.A., Irmis, R.B., Angielczyk, K.D., Smith, R.M.H., and Tsuji, L.A. 2010. Ecologically distinct dinosaurian sister group shows early diversification of Ornithodira. Nature, 464:95-98.

Norman, D.B., Witmer, L.M., and Weishampel, D.B. 2004. Basal Ornithischia, p. 325-334. In Weishampel, D.B., Dodson, P., and Osmólska, H. (eds.), The Dinosauria: Second Edition. University of California Press, Berkeley.
Padian, K. 1990. The ornithischian form genus Revueltosaurus from the Petrified Forest of Arizona (Late Triassic: Norian; Chinle Formation). Journal of Vertebrate Paleontology, 10:268-269.

Parker, W.G., Irmis, R.B., Nesbitt, S.J., Martz, J.W., and Browne, L.S. 2005. The Late Triassic pseudosuchian Revueltosaurus callenderi and its implications of the diversity of early ornithischian dinosaurs. Proceedings of the Royal Society of London B, 272:963-969.

Rensberger, J.M. 1997. Mechanical adaptation in enamel, p. 237-257. In Koenigswald, W.v. and Sander, P.M. (eds.), Tooth enamel microstructure. A.A. Balkema, Rotterdam

Rohlf, F.J. 2010. tpsDig. version 2.0. Downloaded from life.bio.sunysb.edu/morph/

Sander, P.M. 1999. The microstructure of reptilian tooth enamel: terminology, function, and phylogeny. Müncher Geowissenschafliche Abhandlungen Reihe A, 38:1-102.

Sander, P.M. 2000. Prismless enamel in amniotes: terminology, function, and evolution, p. 92-106. In Teaford, M.F., Smith, M.M., and Ferguson, M.W.J. (eds.), Development, function and evolution of teeth. Cambridge University Press, Cambridge.

Sereno, P.C. 1991. Lesothosaurus, "fabrosaurids," and the early evolution of Ornithischia. Journal of Vertebrate Paleontology, 11:168-197.

Smith, J.B. and Dodson, P. 2003. A proposal for a standard terminology of anatomical notation and orientation in fossil vertebrate dentitions. Journal of Vertebrate Paleontology, 23(1):1-12.

Stokosa, K. 2001. Enamel variation within the Theropoda and its biomechanical implications. Journal of Vertebrate Paleontology, 21(supplement to no. 3):105A.

Stokosa, K. 2005. Enamel microstructure variation within the Theropoda, p. 163-178. In Carpenter, K. (ed.), The Carnivorous Dinosaurs. Indiana University Press, Bloomington.

Walker, A.D. 1961. Triassic reptiles from the Elgin area: Stagonolepis, Dasygnathus, and their allies. Philosophical Transactions of the Royal Society of London $B, 244: 103-204$.

Zanno, L.E. and Mackovicky, P. 2011. Herbivorous ecomorphology and specialization patterns in theropod dinosaur evolution. Proceedings of the National Academy of Science, 108:232-237. 